\title{
A multiresolution spatial parameterization for the estimation of fossil-fuel carbon dioxide emissions via atmospheric inversions
}

\author{
J. Ray ${ }^{1}$, V. Yadav ${ }^{2}$, A. M. Michalak ${ }^{2}$, B. van Bloemen Waanders ${ }^{3}$, and S. A. McKenna ${ }^{4}$ \\ ${ }^{1}$ Sandia National Laboratories, P.O. Box 969, Livermore, CA 94551, USA \\ ${ }^{2}$ Carnegie Institution for Science, Stanford, CA 94305, USA \\ ${ }^{3}$ Sandia National Laboratories, P.O. Box 5800, Albuquerque, NM 87185-0751, USA \\ ${ }_{4}^{4}$ IBM Research, Smarter Cities Technology Centre, Bldg 3, Damastown Industrial Estate, Mulhuddart, Dublin 15, Ireland
}

Correspondence to: J. Ray (jairay@ sandia.gov)

Received: 28 December 2013 - Published in Geosci. Model Dev. Discuss.: 6 February 2014

Revised: 2 July 2014 - Accepted: 18 July 2014 - Published: 3 September 2014

\begin{abstract}
The characterization of fossil-fuel $\mathrm{CO}_{2}\left(\mathrm{ffCO}_{2}\right)$ emissions is paramount to carbon cycle studies, but the use of atmospheric inverse modeling approaches for this purpose has been limited by the highly heterogeneous and nonGaussian spatiotemporal variability of emissions. Here we explore the feasibility of capturing this variability using a low-dimensional parameterization that can be implemented within the context of atmospheric $\mathrm{CO}_{2}$ inverse problems aimed at constraining regional-scale emissions. We construct a multiresolution (i.e., wavelet-based) spatial parameterization for $\mathrm{ffCO}_{2}$ emissions using the Vulcan inventory, and examine whether such a parameterization can capture a realistic representation of the expected spatial variability of actual emissions. We then explore whether sub-selecting wavelets using two easily available proxies of human activity (images of lights at night and maps of built-up areas) yields a low-dimensional alternative. We finally implement this low-dimensional parameterization within an idealized inversion, where a sparse reconstruction algorithm, an extension of stagewise orthogonal matching pursuit (StOMP), is used to identify the wavelet coefficients. We find that (i) the spatial variability of fossil-fuel emission can indeed be represented using a low-dimensional wavelet-based parameterization, (ii) that images of lights at night can be used as a proxy for sub-selecting wavelets for such analysis, and (iii) that implementing this parameterization within the described inversion framework makes it possible to quantify fossil-fuel emissions at regional scales if fossil-fuel-only $\mathrm{CO}_{2}$ observations are available.
\end{abstract}

\section{Introduction}

The characterization of fossil-fuel $\mathrm{CO}_{2}\left(\mathrm{ffCO}_{2}\right)$ emissions is paramount to carbon cycle studies. $\mathrm{ffCO}_{2}$ emissions are the largest net carbon flux at the atmosphere-surface interface (Friedlingstein et al., 2006) and spatially disaggregated (or gridded) $\mathrm{ffCO}_{2}$ emissions form a critical input into general circulation and integrated assessment models (Andres et al., 2012). An understanding of fossil-fuel emissions is clearly necessary for characterizing the anthropogenic climate impact. In addition, a process-level understanding of the terrestrial carbon sink requires the quantification of terrestrial biospheric fluxes at fine spatiotemporal scales, which, in turn, requires the differentiation between anthropogenic and biospheric fluxes at those scales.

Gridded inventory estimates of $\mathrm{ffCO}_{2}$ emissions can be derived using socio-economic data (Oda and Maksyutov, 2011; Rayner et al., 2010), and such "bottom-up" estimates have been proposed as a means of monitoring international agreements aimed at mitigating $\mathrm{ffCO}_{2}$ emissions (Pacala et al., 2010). Gridded inventory estimates are derived from $\mathrm{ffCO}_{2}$ budgets and produced by a few institutions; see Andres et al. (2012) for a list. These budgets are compiled from national and provincial statistics on fossil-fuel production and consumption. These large-scale estimates can then be downscaled to finer spatiotemporal scales using easily observed proxies of human activity (and consequently $\mathrm{ffCO}_{2}$ emissions) such as images of lights at night (henceforth "nightlights"), population density, etc. (Oda and Maksyutov, 2011; Rayner et al., 2010; Doll et al., 2000). More sophisticated 
approaches to the fine-scale bottom-up estimation of $\mathrm{ffCO}_{2}$ emissions have also begun to emerge, including, for example, the Vulcan inventory that includes estimates for the US at a $10 \mathrm{~km}$ and hourly resolution for 2002 (http://vulcan.project. asu.edu; Gurney et al., 2009). Such approaches rely on detailed reporting and monitoring data, which are not currently available for many regions of the world.

Although inventory estimates provide a key tool in the understanding of anthropogenic $\mathrm{CO}_{2}$ emissions, their accuracy at large scales depends on the accuracy of reported national consumption data, e.g., the error in $\mathrm{ffCO}_{2}$ emissions from China lies in the 15-20\% range (Gregg et al., 2008). When evaluated at finer spatiotemporal scales, their accuracy also depends on the method used to disaggregate national/provincial $\mathrm{ffCO}_{2}$ emission budgets to finer spatial scales. Pregger et al. (2007) showed that two $0.5^{\circ}$ inventories for Western Europe, for 2003, differed at the grid-cell level by $20 \%$, with a standard deviation of $40 \%$; at finer resolutions, the disagreement worsened. Sources of errors in inventories are discussed in detail in Andres et al. (2012), Raupach et al. (2009) and Rayner et al. (2010). These uncertainties lead to frequent corrections of sub-national inventories of combustion products (Streets et al., 2006) and model predictions of $\mathrm{CO}_{2}$ concentrations that disagree with observations locally (Brioude et al., 2012).

Given both the benefits, and the limitations, of inventorybased estimates, interest has emerged in the development of "top-down" estimates of $\mathrm{ffCO}_{2}$ emissions. These estimates rely on attributing the observed variability in $\mathrm{CO}_{2}$ and related trace gas concentrations in the atmosphere to the underlying fossil-fuel emissions, through the application of statistical atmospheric inversion methods. Some of the proposed approaches, (e.g., Turnbull et al., 2011) have relied on observations of $\Delta^{14} \mathrm{CO}_{2}$ measurements or other non- $\mathrm{CO}_{2}$ tracers. One challenge with these approaches, however, is a combination of the limited number of available observations and the need to understand emission ratios for any co-emitted tracers. Atmospheric inversions relying on atmospheric $\mathrm{CO}_{2}$ measurements, on the other hand, have primarily targeted biospheric $\mathrm{CO}_{2}$ fluxes, often by first pre-subtracting the fossilfuel influence calculated from an inventory; see Ciais et al. (2010) for a review of atmospheric inversion methods. The measurements then consist of $\mathrm{CO}_{2}$ concentration obtained from in situ or remote-sensing observations, and estimates are obtained at a variety of spatiotemporal resolutions for either global or regional domains. The statistical approaches applied typically rely on Gaussian assumptions for flux residuals from prior estimates or other spatiotemporal patterns. Investigations aimed at the estimation of $\mathrm{ffCO}_{2}$ emissions are less common because (1) measurements of $\mathrm{ffCO}_{2}$ concentrations, (e.g., using $\Delta^{14} \mathrm{CO}_{2}$ ) are expensive and not comprehensive and (2) the statistical assumptions used in inversions aimed at understanding biospheric fluxes are ill-suited to the highly heterogeneous and non-Gaussian spatiotemporal variability of $\mathrm{ffCO}_{2}$ emissions. However, some estimates

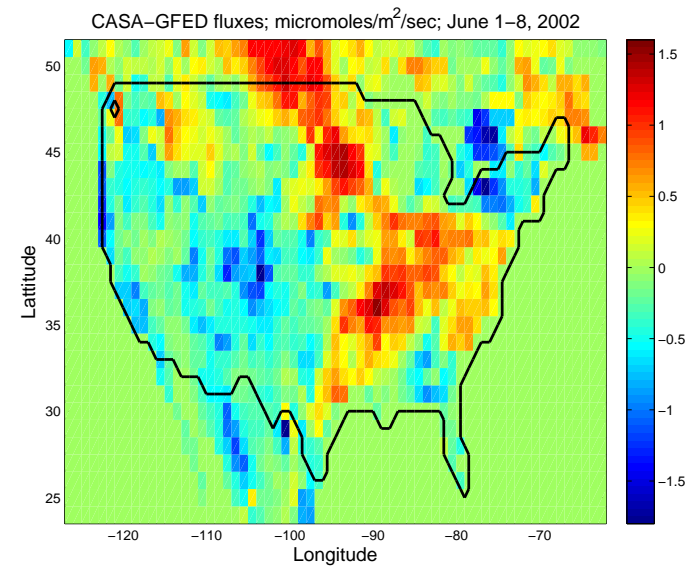

(a) Biospheric fluxes

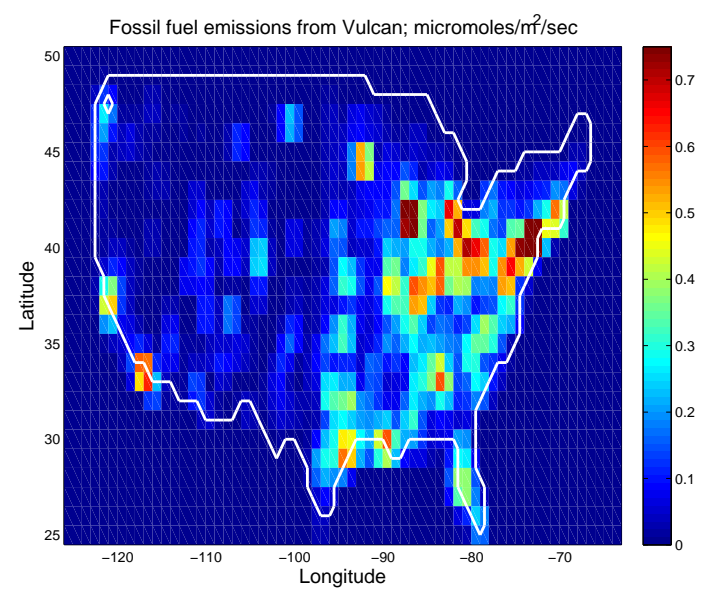

\section{(b) $\mathrm{ffCO}_{2}$ emissions}

Figure 1. Differences in the spatial distribution of biospheric (top) and fossil-fuel (bottom) $\mathrm{CO}_{2}$ fluxes. The biospheric fluxes are stationary, whereas $\mathrm{ffCO}_{2}$ emissions are non-stationary and correlated with human habitation. The fluxes/emissions cover 1-8 June 2002. The biospheric fluxes are obtained from CASA-GFED (http://www. globalfiredata.org/index.html). The post-processing steps to obtain the fluxes as plotted are described in Gourdji et al. (2012). The units of fluxes/emissions are $\mu \mathrm{mol} \mathrm{s}-1 \mathrm{~m}^{-2}$ of $\mathrm{C}$. The $\mathrm{ffCO}_{2}$ emissions are calculated by spatiotemporal averaging of the Vulcan inventory. Note the different color maps; $\mathrm{ffCO}_{2}$ emissions can assume only non-negative values.

of $\mathrm{ffCO}_{2}$ emissions at urban scales are beginning to emerge, including for Salt Lake City (McKain et al., 2012), Indianapolis (Gurney et al., 2012), and Sacramento (Turnbull et al., 2011). 
The goal of the work presented here is to address the second limitation above by exploring the possibility of defining an inversion framework that is specifically targeted at the characteristics of the spatiotemporal variability of $\mathrm{ffCO}_{2}$ emissions at regional scales. We will model spatial variability at $1^{\circ} \times 1^{\circ}$ resolution. Such a framework would require, among other things, a low-dimensional spatial parameterization of $\mathrm{ffCO}_{2}$ emissions, given the data limitations associated with any atmospheric inversion system. We explore this topic through a sequence of three specific objectives:

1. Identification of a low-dimensional parameterization for $\mathrm{ffCO}_{2}$ emissions. $\mathrm{ffCO}_{2}$ emissions are strongly nonstationary (see Fig. 1), and any parameterization must be able to represent such variability. Wavelets, which are an orthogonal basis set with compact support, are widely used to model non-stationary fields, e.g., images (Strang and Nguyen, 1997; Chan and Shen, 2005). We will examine a number of wavelet families to identify the wavelet type that can represent $\mathrm{ffCO}_{2}$ emissions most efficiently, i.e., with minimum error if only a limited number of wavelets were to be retained. The $\mathrm{ffCO}_{2}$ emissions will be obtained from the Vulcan inventory (for the US only) as a realistic example of what the variability of true $\mathrm{ffCO}_{2}$ emissions is likely to be. This objective will ultimately answer the question of whether a low-dimensional parameterization is possible for the type of spatial variability expected in real $\mathrm{ffCO}_{2}$ emissions.

2. Evaluation of the use of a low-dimensional parameterization in combination with easily available proxies of anthropogenic emissions. For most areas of the world, fine-scale estimates of $\mathrm{ffCO}_{2}$ emissions are based on downscaling of national inventories using easily observed proxies of human activity, such as maps of nightlights or of built-up areas (BUA). In this second objective, we will use the wavelet types selected in the first objective, and sub-select them using these two proxies of human activity for the United States. We will then evaluate the degree to which the remaining wavelets can be used to represent the complexity of spatial patterns in $\mathrm{ffCO}_{2}$ emissions. The Vulcan inventory will be used for this purpose too. This objective will answer the question of whether an easily observable proxy can be used to reduce the dimensionality of a wavelet-based spatial parameterization for $\mathrm{ffCO}_{2}$ emission fields. The set of wavelets selected in this manner form a random field model, which we will refer to as the multiscale random field (MsRF) model.

3. Evaluation of the parameterization in an atmospheric inversion, using sparse reconstruction. In the third objective, we will use the reduced basis identified in $\mathrm{Ob}$ jective 2 within an idealized synthetic-data atmospheric inversion aimed at characterizing the spatiotemporal variability of US $\mathrm{ffCO}_{2}$ emissions. A new sparsityenforcing optimization method that preserves the nonnegative nature of $\mathrm{ffCO}_{2}$ emissions will be used to solve the inverse problem. (The terms sparsity and sparsityenforcement are defined in Sect. 2.) The new sparse reconstruction method is used to ensure an unique solution and to guard against overfitting (fitting to observational noise). The optimization procedure will identify the subset of wavelets in the MsRF that can actually be estimated from the observations, while "turning off" the rest. In doing so, it will ensure that the MsRF, as designed, has sufficient flexibility to extract information on $\mathrm{ffCO}_{2}$ from the observations. For simplicity, the synthetic-data inversion will focus only on $\mathrm{ffCO}_{2}$ emissions. We recognize that an ultimate application with real data would require a combination of methods to capture both the biospheric and fossil-fuel signals, or would require the pre-subtraction of the influence of biospheric fluxes on observations. For the purposes of the work presented here, however, the question that we aim to answer is whether an inversion approach based on a low-dimensional parameterization is feasible, even under idealized conditions.

We view this investigation as a methodological first step in the development of an inversion scheme for $\mathrm{ffCO}_{2}$ emissions. To that end, we focus on algorithmic and parameterization issues, and demonstrate our solution in an idealized setting. We use synthetic-data collected from a measurement network sited with an eye towards biospheric $\mathrm{CO}_{2}$ fluxes, as a network optimized for $\mathrm{ffCO}_{2}$ measurement does not exist. We also ignore emissions outside the US. Further, we assume that the errors incurred by the transport model are small, uniform across time and all measurement locations. Thus the method would need to be extended to be used in a realistic setting; we identify some of these adaptations, as well as potential starting points for such investigations. The proposed method, by construction, addresses two issues peculiar to $\mathrm{ffCO}_{2}$ emissions: (1) it is insensitive to underreporting of country-wide $\mathrm{ffCO}_{2}$ emissions; and (2) it can (approximately) capture intense regions of $\mathrm{ffCO}_{2}$ emissions, even when the spatial parameterization is deficient.

The paper is structured as follows. In Sect. 2, we review the use of wavelet modeling in inverse problems. In Sect. 3, we investigate families of wavelets for modeling $\mathrm{ffCO}_{2}$ emissions and construct two MsRF models, based on nightlights and maps of BUA. In Sect. 4, we describe the inverse problem and the numerical method used to solve it. In Sect. 5 we perform inversion tests with synthetic data. In Sect. 6 we discuss the idealizations adopted in this paper and how they may be relaxed. Conclusions are in Sect. 7 . 


\section{Wavelet modeling in inverse problems}

Wavelets are a family of orthogonal bases with compact support (Williams and Amaratunga, 1994; Walker, 2008). They are generated using a scaling function $\phi^{\prime}$ that obeys the recursive relationship: $\phi^{\prime}(x)=\sum_{i} c_{i} \phi^{\prime}(2 x-i)$. A wavelet $\phi$ is generated from the scaling function by taking differences, e.g., $\phi(x)=\sum_{i}(-1)^{i} c_{1-i} \phi^{\prime}(2 x-i)$. The choice of the filter coefficients $c_{i}$ and $\phi^{\prime}$ determine the type of the resulting wavelets. The simplest type is the Haar, which are symmetric, but not differentiable. Wavelets can be shrunk and translated, i.e., $\phi_{s, i}=2^{\frac{s}{2}} \phi\left(2^{s} x-i\right)$, where $s$ is the dilation scale and $i$ refers to translation (location). This allows them to model complex, non-stationary functions efficiently. For each increment in scale, the support of the wavelet halves. Wavelets are defined on dyadic (power-of-two) hierarchical or multiresolution grids.

Consider a domain of size $D$, discretized by a hierarchy of meshes with resolutions $\Delta D / D=\left\{1,1 / 2,1 / 2^{2}, \ldots 1 / 2^{M}\right\}$. Wavelets are defined on each of the levels of the hierarchical mesh and can be positioned at any even-numbered gridcell $2 i, 0 \leq 2 i \leq 2^{s}-1$, on any scale $s$ of the hierarchical mesh. An arbitrary 1-D function $g(x)$ can be represented as $g(x)=w^{\prime} \phi^{\prime}(x)+\sum_{s=1}^{M} \sum_{i=0}^{2^{(s-1)}-1} w_{s, i} \phi_{s, i}(x)$, where the coefficients (or weights) $w_{s, i}$ and $w^{\prime}$ are obtained, via projections of $g(x)$, using fast wavelet transforms. In 2-D, a function $g(x, y)$, defined on a $D \times D$ domain with a hierarchical $2^{M} \times 2^{M}$ mesh, can be subjected to a wavelet transform by applying 1-D wavelet transforms repeatedly, e.g., first by rows and then by columns. Wavelets of scale $s$ have a support $2^{M-s} \times 2^{M-s}, 0 \leq s \leq M$ and can be positioned (in 2-D space) at location $(i, j), 0 \leq(i, j)<2^{s}$. A 2-D wavelet transform results in $2^{M} \times 2^{M}$ wavelet coefficients. In general,

$g(x, y)=w^{\prime} \phi^{\prime}(x, y)+\sum_{s=1}^{M} \sum_{i, j}^{\mathfrak{W}^{(s)}} w_{s, i, j} \phi_{s, i, j}(x, y)$,

where $\mathfrak{W}^{(s)},\left|\mathfrak{W}^{(s)}\right|=\left(4^{s}-4^{s-1}\right)$, is the set of $(i, j)$ indices of wavelet coefficients on scale $s .\left|\mathfrak{W}^{(s)}\right|$ is the size of the set, i.e., the number of wavelets in $\mathfrak{W}^{(s)}$. A large number of fine-scale (i.e., high $s$ ) wavelets model fine spatial details.

\subsection{Wavelet-based random field models}

Random field (RF) models (Cressie, 1993) provide a systematic way of generating multi-dimensional fields based on values assumed by the model's parameters. The parameters are independent and can assume random values, thus generating random fields. Any structure that a field may need to obey, e.g., smoothness, is encoded into the model. RF models may be used for dimensionality reduction, e.g., one can generate fields on a fine grid by varying a handful of parameters. Alternatively, one can design a RF model to generate fields systematically, by independently varying parameters that control structures at different spatial scales. The spatial parameteri- zation that we construct in Sect. 3 is an instance of the latter type of RF model.

Wavelets are often used to represent fields, most commonly, to represent images, e.g., the JPEG2000 standard (Taubman and Marcellin, 2002). Most fields/images are compressible in a judiciously chosen wavelet basis, i.e., most of the wavelet coefficients are small and can be discarded to form a reduced-rank approximation of the field (Welstead, 1999). In Auger and Tangborn (2002), a reduced-rank wavelet model was developed for global, time-variant $\mathrm{CH}_{4}$ concentration, which were updated with limited observations using a Kalman filter. The selection of wavelets to form the random field (RF) model was done empirically, by decimating the fine-scale wavelets. The construction of the RF model can be performed more rigorously if a prior model is available. In Jafarpour (2011) wavelets were used in the reconstruction of permeability fields from limited measurements of flow through a porous medium. An ensemble of permeability field realizations, drawn from the prior distribution, were used to develop a multivariate Gaussian prior distribution for the wavelet coefficients. The RF model was created by discarding wavelet coefficients with small means. In Romberg et al. (2001), the authors constructed a hidden Markov model to encode the relationship between the wavelet coefficients on adjacent scales of the wavelet tree. This RF model was used to reconstruct compressively sensed signals (Duarte et al., 2008) and images (He and Carin, 2009). Thus the use of wavelet-based RF models to parameterize and reconstruct complex fields from limited measurements is quite common.

The RF model need not be constructed offline using a prior; it can also be constructed during the inversion, in a data-driven manner. This occurs in the compressive sensing (CS) of signals and images (Candes and Wakin, 2008). In this approach, all the wavelets in a field's representation are retained and the ones that cannot be estimated from available observations are identified and removed. Let $\mathbf{g}$ be an image of size $N$ that can be represented sparsely using $L \ll N$ wavelets. Let $\mathbf{g}^{\prime}$, of size $N_{\mathrm{m}}, L<N_{\mathrm{m}} \ll N$, be its compressed measurement, obtained by projecting $\mathbf{g}$ onto a set of random vectors $\boldsymbol{\psi}_{i}$, i.e., $\mathbf{g}^{\prime}=\boldsymbol{\Psi} \mathbf{g}=\boldsymbol{\Psi} \boldsymbol{\Phi} \mathbf{w}$. Here the rows of $\boldsymbol{\Psi}$ consist of the random vectors $\boldsymbol{\psi}_{j}$ and columns of $\boldsymbol{\Phi}$ consist of wavelets $\phi_{i}$. $\boldsymbol{\Phi}$ is a $N \times N$ matrix while $\boldsymbol{\Psi}$ is $N_{\mathrm{m}} \times N$. The bulk of the theory that allows reconstruction of the image/field with such observations was established in Candes and Tao (2006), Donoho (2006), and Candes et al. (2006). In CS, the reconstruction of $\mathbf{g}$ (alternatively $\mathbf{w}$ ) can be performed using a number of methods. It is posed as an optimization problem

$\underset{\mathbf{w} \in \mathbb{R}^{N}}{\operatorname{minimize}}\|\mathbf{w}\|_{1}$, subject to $\left\|\mathbf{g}^{\prime}-\mathbf{A w}\right\|_{2}<\epsilon_{2}, \quad \mathbf{A}=\boldsymbol{\Psi} \boldsymbol{\Phi}$. 
Thus we enforce sparsity in $\mathbf{w}$ with its $\ell_{1}$ norm $\left(\|\cdot\|_{1}\right)$, under the constraint that the $\ell_{2}$ norm $\left(\|\cdot\|_{2}\right)$ of the misfit between $\mathbf{g}^{\prime}$ and the image reconstructed from $\mathbf{w}$ is kept within a bound. Some of the methods to solve this problem are basis pursuit (Chen et al., 1998), matching pursuit (Mallat and Zhang, 1993), orthogonal matching pursuit (Tropp and Gilbert, 2007) and stagewise orthogonal matching pursuit (StOMP; Donoho et al., 2012). We will refer to the process of enforcing sparsity as "sparsification" and the result of the process will be called the "sparsified" or sparse solution.

\subsection{Wavelets and sparsity in inverse problems}

Sparsity is often used to solve inverse problems in physics, with the $\boldsymbol{\Psi}$ operator representing the physical process. In $\mathrm{Li}$ and Jafarpour (2010), sparsity was used to estimate a permeability field (represented by wavelets) from fluid transport observations. A good review of the use of sparsity in permeability field reconstruction is available in Jafarpour (2013). Seismic tomography, which estimates subsurface geologic facies, also has exploited sparsity for reconstruction. This has been demonstrated with wavelet representations of the subsurface and nonlinear forward models (Loris et al., 2007; Simons et al., 2011). Gholami and Siahkoohi (2010) used a split Bregman iteration (Goldstein and Osher, 2009) to solve a seismic tomography problem, imposing sparsity via soft thresholding (Donoho, 1995). The authors also expanded the imposition of sparsity from the wavelet space to the finite-difference space, i.e., they used an $\ell_{1}$ norm to sparsify deviations of the solution from a "best-guess", in the absence of constraining observations. In this manner, both the prior/guessed solution and sparsity are used to regularize the inversion.

To summarize, wavelet-based RF models are routinely used in inverse problems. Their dimensionality can be reduced a priori using prior information. Data-driven dimensionality reduction can also be performed by enforcing sparsity during the inversion. This has been demonstrated with nonlinear problems too.

\section{Constructing a multiscale random field model}

We seek an approximate representation of $\mathrm{ffCO}_{2}$ emissions, which is low dimensional or sparse, i.e., many of the $w_{s, i, j}$ in Eq. (1) may be set to zero. For this purpose, we use $\mathrm{ffCO}_{2}$ emissions from the Vulcan inventory, coarsened to $1^{\circ}$ resolution and averaged over a year to yield $\overline{\mathbf{f}_{\mathrm{V}}}$ (see Fig. 1 for a plot). The emissions are described on a $2^{M} \times 2^{M}$ grid, $M=6$. The finest wavelets, on scale $s=6$, have a support of $2^{\circ} \times 2^{\circ}$; the ones on $s=5$ are a factor of two bigger, i.e., they have a support of $4^{\circ} \times 4^{\circ}$. The rectangular domain extents are given by the corners $24.5^{\circ} \mathrm{N}, 63.5^{\circ} \mathrm{W}$ and $87.5^{\circ} \mathrm{N}$, $126.5^{\circ} \mathrm{W}$. $\mathrm{ffCO}_{2}$ emissions are restricted to $\mathcal{R}$, the lower 48 states of the US.
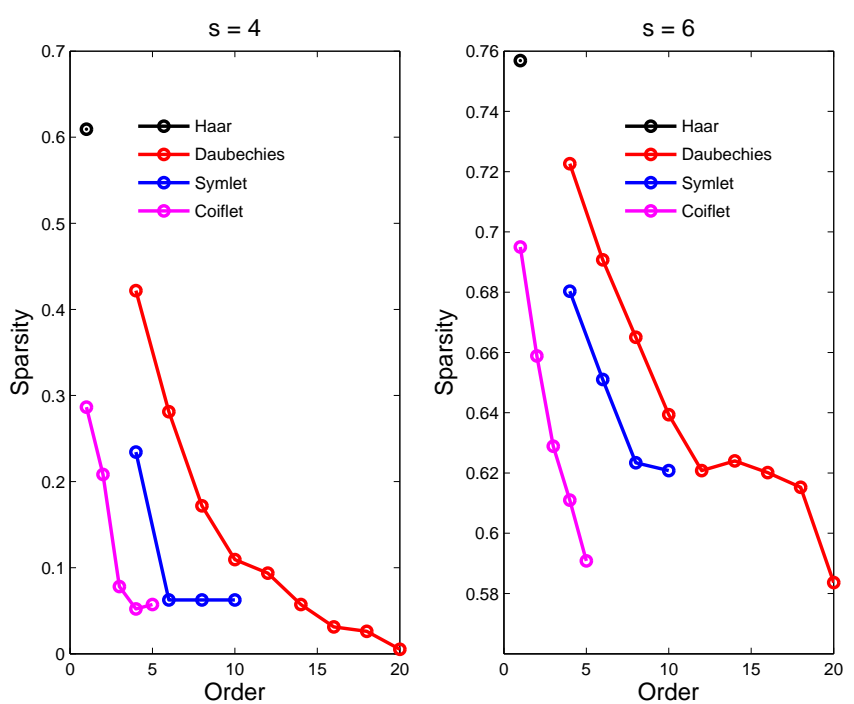

Figure 2. Sparsity of representation at scale $s=4$ (left) and $s=6$ (right) for a combination of wavelet families and orders. We find that Haar provide the sparsest representation.

\subsection{Choosing a wavelet}

We investigate a number of wavelet families (Haar, Daubechies, Symlet, and Coiflet) in order to determine which provides the sparsest representation of $\overline{\mathbf{f}_{\mathrm{V}}} \cdot \overline{\mathbf{f}_{\mathrm{V}}}$ is first subjected to a wavelet transform using a chosen wavelet. At each scale $s$, we remove wavelets that contribute little to $\overline{\mathbf{f}_{\mathrm{V}}}$. We set "small" wavelet coefficients ("small" is defined as the ratio $\left|w_{s, i, j} / w_{\max , s}\right| \leq 10^{-3}$, where $w_{\max , s}$ is the wavelet coefficient on scale $s$ with the largest magnitude) to zero. We refer to the fraction of zero wavelet coefficients at scale $s$ as its sparsity. Figure 2 plots the sparsity at scale $s=4,6$ for a large combination of wavelet families and orders. We see that the Haar wavelet (also called the Daubechies, order 2 wavelet) provides the sparsest representation, making it a candidate for developing a low-dimensional MsRF for $\mathrm{ffCO}_{2}$ emissions. This is a consequence of the spatial distribution of $\overline{\mathbf{f}_{\mathrm{V}}}-$ the map of $\overline{\mathbf{f}_{\mathrm{V}}}$ (Fig. 1b) is largely empty west of $100^{\circ} \mathrm{W}$, which manifests itself as small coefficients for the wavelets whose support lie in that region. This favors simpler (non-smooth) and low-order wavelets.

Next we investigate the variation of the magnitude of wavelet coefficients, as a function of the type of wavelets used to model $\overline{\mathbf{f}_{\mathrm{V}}}$. We select five wavelet types, e.g., Haar, Daubechies, order 4 and 8, and Symlet, order 4 and 6, and perform a wavelet transform of $\overline{\mathbf{f}_{\mathrm{V}}}$. At each scale $s$, we set the "small" wavelet coefficients to zero. In Fig. 3 we plot the average and standard deviation of the non-zero wavelet coefficients. The means of the wavelet coefficients at the finer scales are small, regardless of the wavelet type. We see that while Haar provide the sparsest representation, the non-zero wavelet coefficients tend to have large magnitudes. In contrast, smoother wavelets with broader support, e.g., 


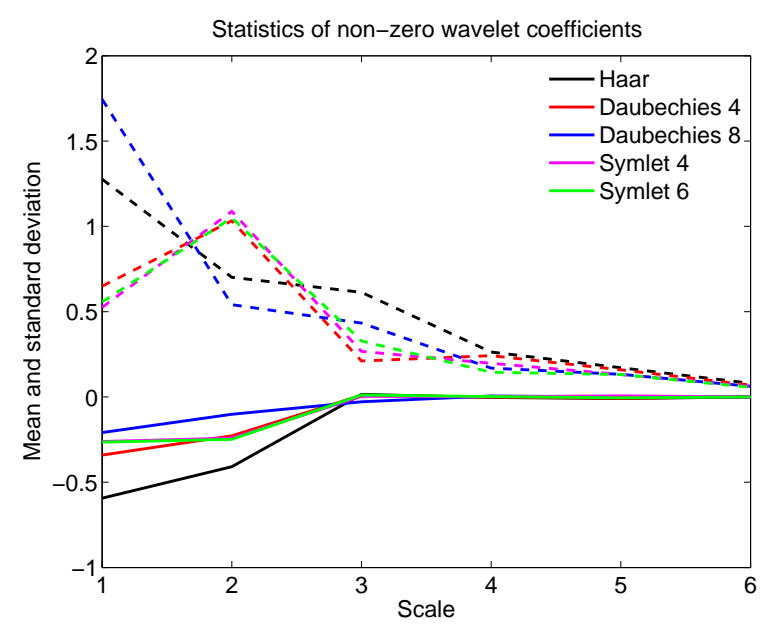

Figure 3. We plot the average value of the non-zero coefficients (solid lines) and their standard deviation (dashed line), at different scales $s$, when $\overline{\mathbf{f}_{\mathrm{V}}}$ is subjected to wavelet transforms using Haar, Daubechies 4 and 6, and Symlet 4 and 6 wavelets. We find that while Haar may provide the sparsest representation (Fig. 2), the non-zero values tend to be large and distinct.

Daubechies, order 8, have more non-zero wavelet coefficients, but with smaller wavelet coefficients. This is a consequence of the spatial distribution of $\overline{\mathbf{f}_{\mathrm{V}}}$ (Fig. 1) which has sharp gradients, placing smooth wavelets at a disadvantage. In Fig. 3, we also see that the means and standard deviations shrink, especially after scale $s=3$; further, the distributions of wavelet coefficients arising from the different wavelet types begin to resemble each other. This arises from the fact that there are sharp boundaries around the areas where $\mathrm{ffCO}_{2}$ emissions occur; when subjected to a wavelet transform, the region in the vicinity of a sharp boundary gives rise to large wavelet coefficients down to the finest scale. Thus the few non-zero wavelet coefficients at the finer scales assume similar values, irrespective of the wavelet type.

Finally, we check the accuracy of a Haar representation of $\overline{\mathbf{f}_{\mathrm{V}}}$ at various levels of sparsity. Again, we define a "small" wavelet as $\left|w_{s, i, j} / w_{\max , s}\right| \leq \alpha$. We perform a wavelet transform of $\overline{\mathbf{f}_{\mathrm{V}}}$ using Haar wavelets and sparsify (set small wavelets to zero) using $10^{-6} \leq \alpha \leq 1$. We then perform an inverse transform to reconstruct a "sparsified" $\overline{\mathbf{f}}_{\mathrm{V}}$. In Fig. 4, we plot overall sparsity, reconstruction error $\epsilon=$ $\left\|\overline{\mathbf{f}_{\mathrm{V}}} ;-\overline{\mathbf{f}_{\mathrm{V}}}\right\|_{2} /\left\|\overline{\mathbf{f}_{\mathrm{V}}}\right\|_{2}$ and the Pearson correlation between the true and reconstructed $\overline{\mathbf{f}_{\mathrm{V}}}$ as a function of $\alpha$. We define the Pearson correlation between $\overline{\mathbf{f}_{\mathrm{V}}}{ }^{\prime}$ and $\overline{\mathbf{f}_{\mathrm{V}}}$ as

$\rho\left(\overline{\mathbf{f}_{\mathrm{V}}}, \overline{\mathbf{f}_{\mathrm{V}}}\right)=\frac{\operatorname{cov}\left(\overline{\mathbf{f}_{\mathrm{V}}}, \overline{\mathbf{f}_{\mathrm{V}}}\right)}{\sigma_{\overline{\mathbf{f}_{\mathrm{V}}}} \sigma_{\overline{\mathbf{f}_{\mathrm{V}}}}^{\prime}}$,

where $\sigma_{\overline{\mathbf{f}_{\mathrm{V}}}}^{2}$ and $\sigma_{\overline{\mathbf{f}_{\mathrm{V}}}}^{2}$ are the variances of the true and reconstructed fluxes and $\operatorname{cov}\left(Z_{1}, Z_{2}\right)$ is the covariance between two random variables $Z_{1}$ and $Z_{2}$. Here $\|\cdot\|_{2}$ denotes the $\ell_{2}$ norm. We find that for $\alpha<10^{-2}$ there is practically no error

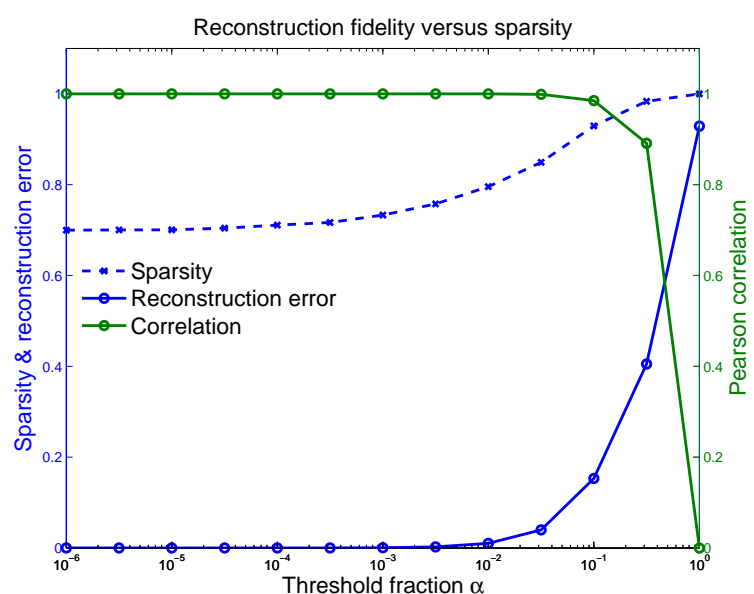

Figure 4. Variation of sparsity, reconstruction error $\epsilon$, and the Pearson correlation between the true and reconstructed $\overline{\mathbf{f}_{\mathrm{V}}}$, i.e., $\rho\left(\overline{\mathbf{f}_{\mathrm{V}}}, \overline{\mathbf{f}}_{\mathrm{V}}^{\prime}\right)$ as a function of $\alpha$.

(as measured by these metrics) though we achieve a sparsification of about $80 \%$. Even at a sparsity of around $90 \%$, the error is less that $10 \%$. Thus a small collection of Haar wavelets have the ability to reproduce $\overline{\mathbf{f}_{\mathrm{V}}}$ with an acceptable degree of error. This low-dimensional character of a Haar representation of $\overline{\mathbf{f}_{\mathrm{V}}}$ can be invaluable in an inverse problem predicated on sparse observations, and henceforth, we will proceed with Haar wavelets as the basis set of choice for representing $\mathrm{ffCO}_{2}$ emissions. Figure 5 shows the decomposition of $\overline{\mathbf{f}_{\mathrm{V}}}$ on a $M=6$ hierarchy of Haar wavelets.

We will use wavelets selected using the (single) nightlight and BUA maps to estimate weekly $\mathrm{ffCO}_{2}$ emissions. Our tests above show that they model annually averaged Vulcan emissions adequately, and we assume that while emissions may wax and wane with time, their spatial distribution does not vary sufficiently to require a new wavelet selection. We base this assumption on $\mathrm{ffCO}_{2}$ emissions' correlation with human activities and static sources like powerplants which do not display large spatial dislocations with time. Note that the location and strengths of intense sources of $\mathrm{ffCO}_{2}$ emissions, such as powerplants, can be found at the CARMA (Carbon Monitoring for Action; website http://carma.org). Note, also, that CARMA is not peer-reviewed and only provides data for a limited number of years (version 3.0 has data only for 2004 and 2009).

\subsection{Constructing a random field model}

We seek a spatial parameterization for $\mathrm{ffCO}_{2}$ emissions, of the form

$\mathbf{f}=w^{\prime} \phi^{\prime}+\sum_{s=1}^{M} \sum_{i, j} w_{s, i, j} \phi_{s, i, j}, \quad\{s, i, j\} \in W^{(s)}$,

where $W^{(s)}$ is a set containing a small number of Haar bases. We will investigate the usefulness of an 

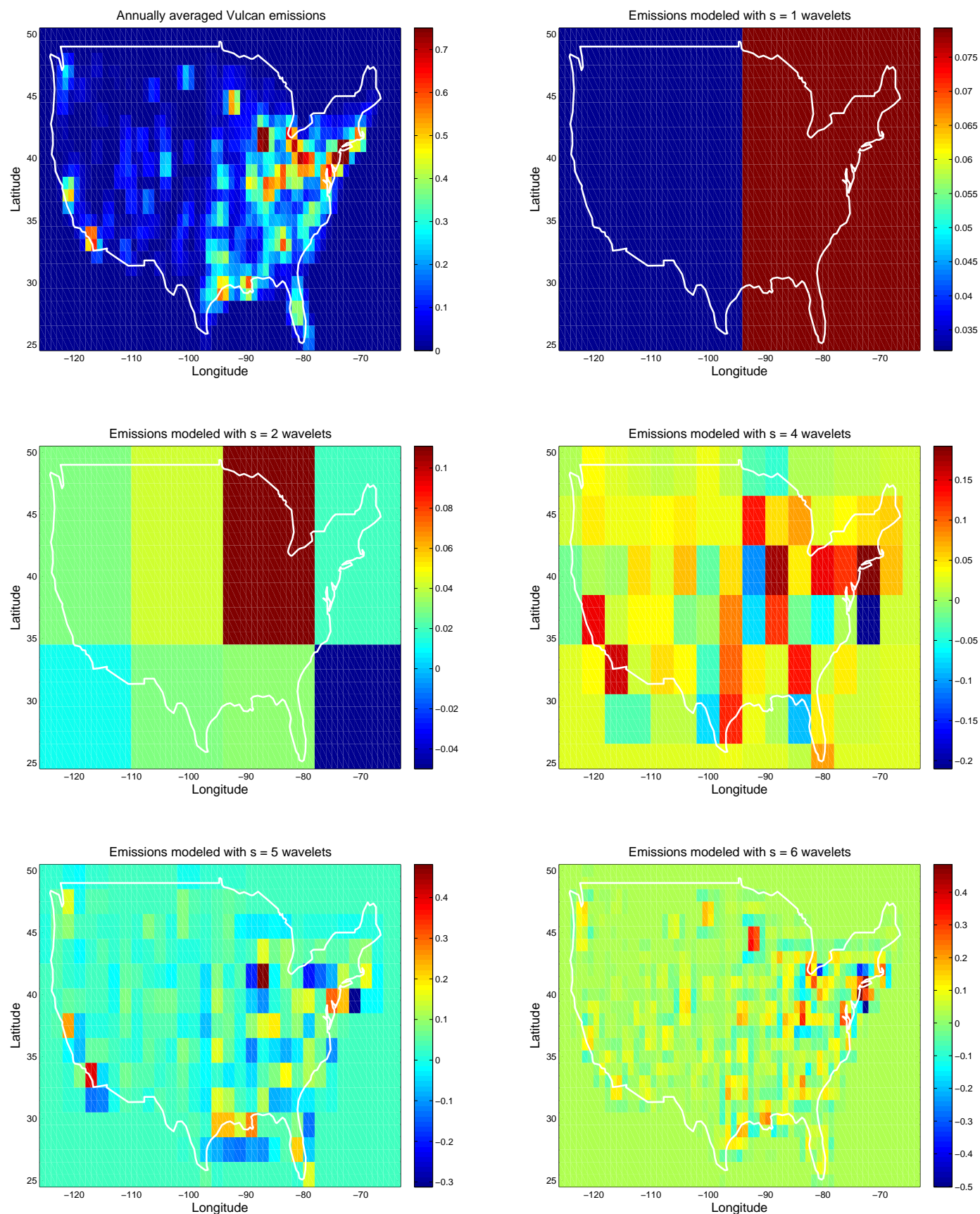

Figure 5. Annually averaged Vulcan emissions $\overline{\mathbf{f}_{\mathrm{V}}}$ are modeled using Haar wavelets on scales 1, 2, 4, 5, and 6. The figure at the top (left) plots $\overline{\mathbf{f}_{\mathrm{V}}}$, and the rest its decomposition across wavelet scales. Note that we have displayed only the relevant part of the dyadic $2^{M} \times 2^{M}$ grid on which wavelets are described.

easily observed proxy $\mathbf{X}$ of human activity (which correlates with $\mathrm{ffCO}_{2}$ emissions) to select the components of $W^{(s)}$. Radiance calibrated nightlights (http://www.ngdc.noaa.gov/dmsp/download_radcal.html;

Cinzano et al., 2000) have been used for constructing $\mathrm{ffCO}_{2}$ inventories (Doll et al., 2000) and are an obvious choice for $\mathbf{X}$. However, nightlight radiances are also affected by economic factors (Raupach et al., 2009), and we will explore maps of built-up area as an alternative (http://www.sage.wisc.edu/atlas/maps.php?datasetid= $18 \&$ includerelatedlinks $=1 \&$ dataset $=18$; B. Miteva, personal communication, 2013). The map of BUA uses nightlight radiances in its computations, and so these are not independent proxies; however, the BUA map also includes 
information from IGBP (International Geosphere-Biosphere Programme, http://www.igbp.net/) land-cover map. The two choices for $\mathbf{X}$ will be compared with respect to (1) sparsity, (2) the correlation between $\mathbf{X}$ and $\overline{\mathbf{f}_{\mathrm{V}}}$, and (3) the ability of $W^{(s)}$ to capture $\overline{\mathbf{f}_{\mathrm{V}}}$.

In Fig. 6 (top row), we plot maps of the two proxies, coarsened to $1^{\circ}$ resolution. Comparing with Fig. 1 (bottom), we see that they bear a strong resemblance to $\overline{\mathbf{f}_{\mathrm{V}}}$. We subject $\mathbf{X}$ to a wavelet transform and set all wavelet coefficients $\left|w_{s, i, j}\right|<$ $\delta$ to zero, where $\delta$ is a user-defined threshold. The bases with non-zero coefficients are selected to constitute $W^{(s)}$. We reconstitute a "sparsified" proxy, $\mathbf{X}^{(s)}$, using just the bases in $W^{(s)}$, and compute the correlation between $\mathbf{X}^{(s)}$ and $\overline{\mathbf{f}_{\mathrm{V}}}$. Finally, we project $\overline{\mathbf{f}_{\mathrm{V}}}$ onto $W^{(s)}$, obtain its "sparsified" form $\overline{\mathbf{f}_{\mathrm{V}}}(s)$, and compute the error $\epsilon_{f}=\left\|\overline{\mathbf{f}_{\mathrm{V}}}(s)-\overline{\mathbf{f}_{\mathrm{V}}}\right\|_{2} /\left\|\overline{\mathbf{f}_{\mathrm{V}}}\right\|_{2}$. In Fig. 6 (middle row), we plot the sparsity, correlation and $\epsilon_{f}$ for various values of $\delta$. We do so for both nightlights and BUA. For nightlights, we achieve a sparsity of around 0.75 for $\delta<10^{-2}$, i.e., we need to retain only a quarter of the Haar bases to represent nightlights. The nightlights so represented bear a correlation of around 0.7 with $\overline{\mathbf{f}_{\mathrm{V}}}$, and achieve an error $\epsilon_{f}$ of around 0.1. Note that this measure of error reflects the inability of the MsRF to represent fine-scale details, and not spatially aggregated quantities, which are represented more accurately. In contrast, using BUA as a proxy, we see that while the sparsity achieved is similar, the correlation between $\mathbf{X}^{(s)}$ and $\overline{\mathbf{f}_{\mathrm{V}}}$ is slightly higher. The behavior of $\epsilon_{f}$ is similar, except the error increases faster with $\delta$, as compared to nightlights. However both nightlights and BUA maps show significant correlation with $\overline{\mathbf{f}_{\mathrm{V}}}$ and the sparsified set of Haar bases that they (i.e., the proxies) provide (using $\delta=10^{-2}$ in both the cases) allow us to construct a low-dimensional parameterization of $\mathrm{ffCO}_{2}$ emissions.

Finally, we use $\mathbf{X}^{(s)}$ to create a "prior model" $\mathbf{f}_{\mathrm{pr}}=c \mathbf{X}^{(s)}$ for $\mathrm{ffCO}_{2}$ emissions, $\mathbf{f}$. is computed such that

$$
\begin{aligned}
& \int_{\mathcal{R}} \overline{\mathbf{f}_{\mathrm{V}}} \mathrm{d} A=\int_{\mathcal{R}} \mathbf{f}_{\mathrm{pr}} \mathrm{d} A= \\
& c \int_{\mathcal{R}}\left(w_{(X)}^{\prime} \phi^{\prime}+\sum_{l, i, j} w_{(X), s, i, j} \phi_{l, i, j}\right) \mathrm{d} A, \quad\{l, i, j\} \in W^{(s)}
\end{aligned}
$$

where $\mathcal{R}$ denotes the lower 48 states of USA and $\mathbf{w}_{(X)}=$ $\left\{w_{(X), s, i, j}\right\}$ are coefficients from a wavelet transform of $\mathbf{X}$. This implies that $c$ is calculated such that both $\overline{\mathbf{f}_{\mathrm{V}}}$ and $\mathbf{f}_{\mathrm{pr}}$ provide the same value for the total emissions for the US. $c$ is the ratio of the aggregate total of $\mathrm{ffCO}_{2}$ emissions to the aggregate total of radiances (for the nightlights) or percentages of built-up areas. In Fig. 6 (bottom row), we plot the error $\left(\mathbf{f}_{\mathrm{pr}}-\overline{\mathbf{f}_{\mathrm{V}}}\right)$. (The Supplement contains a scatter plot of $\mathbf{f}_{\mathrm{pr}}$ vs. $\overline{\mathbf{f}}_{\mathrm{v}}$.) We see that neither nightlights nor the BUA map provide a $\mathbf{f}_{\mathrm{pr}}$ that is an accurate representation of $\overline{\mathbf{f}_{\mathrm{V}}}$, though they share similar spatial patterns, i.e., $\mathbf{f}_{\mathrm{pr}}$ may be used to provide a guess for $\mathbf{f}$ in an inverse problem, but, by itself, is a poor predictor, regardless of the proxy $\mathbf{X}$ used to create it.

\section{Formulation of the estimation problem}

In this section, we pose and solve an inverse problem to estimate $\mathrm{ffCO}_{2}$ emissions using the MsRF developed in Sect. 3. The method is new, and uses a sparse reconstruction method that is summarized in Sects. 4.1 and 4.2; full details are in Ray et al. (2013). The inversion technique is most relevant in situations where accurate, finely gridded $\mathrm{ffCO}_{2}$ inventories are unavailable, and one has to take recourse to easily observable proxies for information on the spatial pattern of $\mathrm{ffCO}_{2}$ emissions.

The inverse problem is predicated on synthetic observations, $\mathbf{y}^{\text {obs }}$, of $\mathrm{CO}_{2}$ concentrations measured at 35 towers (a network that existed in 2008). These are plotted as markers in Fig. 8; see Ray et al. (2013) for their precise locations and names. The measurements are related to $\mathrm{ffCO}_{2}$ emissions described on a finely gridded domain as

$\mathbf{y}^{\text {obs }}=\mathbf{y}+\epsilon=\mathbf{H f}+\epsilon$,

where $\mathbf{H}$ is the transport or sensitivity matrix, obtained from a transport model, $\mathbf{y}$ is the $\mathrm{CO}_{2}$ concentration predicted by the atmospheric model, which differs from its measured counterpart by an error $\epsilon$. The $\mathrm{ffCO}_{2}$ emissions $\mathbf{f}$ are defined on a grid, and are assumed to be non-zero only within $\mathcal{R}$.

The estimation of $\mathrm{CO}_{2}$ fluxes, typically biospheric (Nassar et al., 2011; Chatterjee et al., 2012; Gourdji et al., 2012), is usually posed as the minimization of an objective function $J$,

$J=\left(\mathbf{y}^{\mathrm{obs}}-\mathbf{H} \mathbf{f}\right)^{\mathrm{T}} \mathbf{R}_{\mathrm{e}}^{-1}\left(\mathbf{y}^{\mathrm{obs}}-\mathbf{H} \mathbf{f}\right)+\left(\mathbf{f}-\mathbf{f}_{\mathrm{m}}\right)^{\mathrm{T}} \mathbf{Q}^{-1}\left(\mathbf{f}-\mathbf{f}_{\mathrm{m}}\right)$,

where $\mathbf{f}_{\mathrm{m}}$ are "prior" (or guessed) fluxes and $\mathbf{R}_{\mathrm{e}}^{-1}$ is a diagonal matrix containing the standard deviation of Gaussian noise used to model measurement error. The discrepancy between the "true" and prior fluxes is modeled as a multivariate Gaussian field, whose covariance $\mathbf{Q}$ is calculated offline. In contrast, in our $\mathrm{ffCO}_{2}$ inversion, $\mathbf{f}$ will be modeled using the MsRF rather than a multivariate Gaussian field. Further, the second term in Eq. (6) is omitted and the effect of the "guessed" or "prior" emissions $\mathbf{f}_{\text {pr }}$ is introduced in a manner that is amenable to sparse reconstruction (see Sect. 4.1). The calculation of the sensitivities $\mathbf{H}$ is described in detail in Gourdji et al. (2012); we have reused them in our work. The elements of the $\mathbf{H}$ matrix are calculated using the Stochastic Time-Inverted Lagrangian Transport Model (Lin et al., 2003), with wind fields from the Weather Research \& Forecasting model (Skamarock and Klemp, 2008), version 2.2, driven by 2008 meteorology. Details of the WRF settings and the nested grid used for the wind fields to calculate $\mathbf{H}$ are in Gourdji et al. (2012). Concentration footprints (or sensitivities) were calculated at $3 \mathrm{~h}$ intervals by 

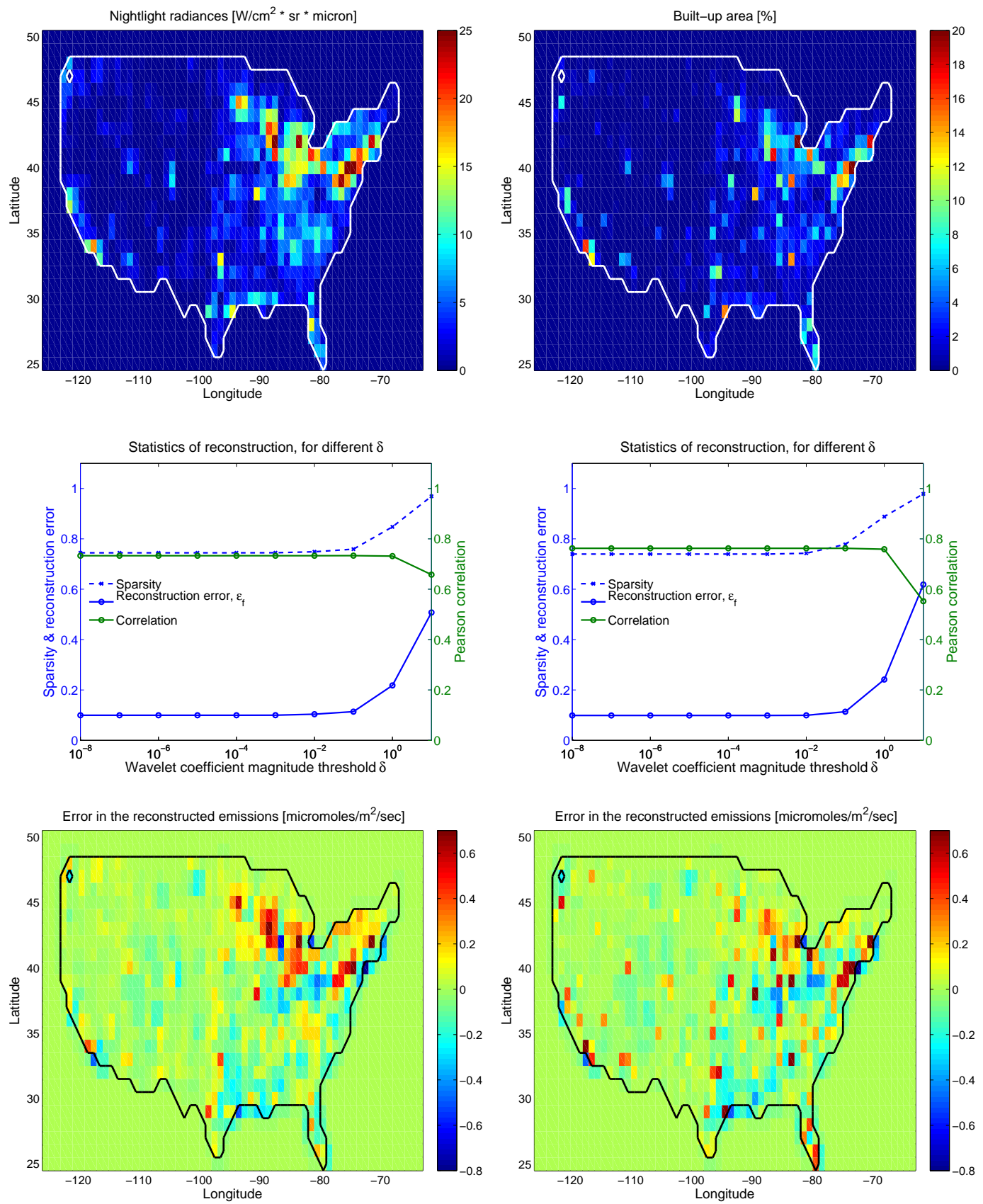

Figure 6. Top row: maps of nightlight radiances (left) and BUA percentage (right), for the US. Middle row: the sparsity of representation, the correlation between $\mathbf{X}$ and $\overline{\mathbf{f}_{\mathrm{V}}}$ and the normalized error $\epsilon_{f}$ between the Vulcan emissions $\overline{\mathbf{f}_{\mathrm{V}}}$ and the sparsified form obtained by projecting it on $\mathbf{X}$. These values are plotted for nightlights (left) and the BUA maps (right). Bottom row: plots of $\left(\mathbf{f}_{\mathrm{pr}}-\overline{\mathbf{f}_{\mathrm{V}}}\right)$ obtained from nightlights (left) and BUA maps (right).

integrating the trajectories over a North American $1^{\circ} \times 1^{\circ}$ grid as described in Lin et al. (2003). The sensitivity of the $\mathrm{CO}_{2}$ concentration at each observation location due to the flux at each grid cell (the "footprint") is calculated in units of ppmv $\mu \mathrm{mol}^{-1} \mathrm{~m}^{2} \mathrm{~s}$ (ppmv: parts per million by volume). $\mathrm{ffCO}_{2}$ emissions were averaged over 8-day intervals and the sensitivity of $\mathbf{y}$ to the 8 day-averaged emissions were obtained from the $3 \mathrm{~h}$ sensitivities described above by simply adding the $8 \times 24 / 3=64$ sensitivities that span the 8 -day period. Thereafter, the grid cells outside $\mathcal{R}$ were removed to obtain the $\mathbf{H}$ matrix used in this study. The size of the $\mathbf{H}$ matrix is $\left(K_{\mathrm{S}} N_{\mathrm{s}}\right) \times\left(N_{\mathcal{R}} K\right)$, where $K_{\mathrm{S}}$ is the number of tower measurements per year, $N_{\mathrm{s}}$ is the number of sensors/towers, $N_{\mathcal{R}}$ is the number of grid cells in $\mathcal{R}$, the part of the domain 
covered by the lower 48 states of the US and $K$ is the number of 8-day periods that constitute the duration over which the emissions are estimated.

\subsection{Posing and solving the inverse problem}

We denote the spatial distribution of emissions during an arbitrary 8-day period $k$ as $\mathbf{f}_{k}$. The 8-day period was chosen to minimize aggregation error. We seek emissions over an entire year, i.e., we seek $\mathbf{F}=\left\{\mathbf{f}_{k}\right\}, k=1 \ldots K$. We will model emissions on the $2^{M} \times 2^{M}, M=6$ mesh with wavelets:

$$
\begin{aligned}
\mathbf{f}_{k} & =w_{k}^{\prime} \phi^{\prime}+\sum_{s=1}^{M} \sum_{i, j} w_{s, i, j, k} \phi_{s, i, j}, \quad\{s, i, j\} \in W^{(s)} \\
& =\boldsymbol{\Phi} \mathbf{w}_{k} .
\end{aligned}
$$

Note that $\boldsymbol{\Phi}$ comprises of only those wavelets selected using $\mathbf{X}$ and contained in $W^{(s)}$. For the entire year, the expression for emissions becomes $\mathbf{F}=\left\{\mathbf{f}_{1}, \mathbf{f}_{2}, \ldots \mathbf{f}_{K}\right\}=$ $\left\{\boldsymbol{\Phi} \mathbf{w}_{1}, \boldsymbol{\Phi} \mathbf{w}_{2}, \ldots \boldsymbol{\Phi} \mathbf{w}_{K}\right\}=\widetilde{\boldsymbol{\Phi}} \mathbf{w}$. Since $\boldsymbol{\Phi} \mathbf{w}_{k}$ models the emissions over all grid cells, i.e., over the rectangular region given by the corners $\left(24.5^{\circ} \mathrm{N}, 63.5^{\circ} \mathrm{W}\right)$ and $\left(87.5^{\circ} \mathrm{N}, 126.5^{\circ} \mathrm{W}\right)$, and not just $\mathcal{R}, \mathbf{F}$ contains emissions over the lower 48 states, as well as the region outside it (where we have assumed that the emissions are non-existent). We separate out the two fluxes by permuting the rows of $\widetilde{\boldsymbol{\Phi}}$

$\mathbf{F}=\left(\begin{array}{c}\mathbf{F}_{\mathcal{R}} \\ \mathbf{F}_{\mathcal{R}^{\prime}}\end{array}\right)=\left(\begin{array}{c}\widetilde{\boldsymbol{\Phi}}_{\mathcal{R}} \\ \widetilde{\boldsymbol{\Phi}}_{\mathcal{R}^{\prime}}\end{array}\right) \mathbf{w}$,

where $\widetilde{\boldsymbol{\Phi}}_{\mathcal{R}}$ and $\widetilde{\boldsymbol{\Phi}}_{\mathcal{R}^{\prime}}$ are $\left(N_{\mathcal{R}} K\right) \times(L K)$ and $\left(N_{\mathcal{R}^{\prime}} K\right) \times(L K)$ matrices, respectively. Here $L$ is the number of wavelets in $W^{(s)}$ and $N_{\mathcal{R}^{\prime}}$ is the number of grid cells in $\mathcal{R}^{\prime}$, the region outside $\mathcal{R}$ but inside the rectangular domain. The modeled concentrations at the measurement towers, caused by $\mathbf{F}_{\mathcal{R}}$, can be written as $\mathbf{y}=\mathbf{H F}_{\mathcal{R}}$. For arbitrary $\mathbf{w}, \mathbf{F}_{\mathcal{R}^{\prime}}$, the emissions in the region outside $\mathcal{R}$, are not zero. Consequently, it will be necessary to specify $\mathbf{F}_{\mathcal{R}^{\prime}}=0$ as a constraint in the inverse problem.

Specifying the constraint $\mathbf{F}_{\mathcal{R}^{\prime}}=0$ directly is not very efficient since it leads to $N_{\mathcal{R}^{\prime}} K$ constraints. In a global inversion, or at resolutions higher than $1^{\circ} \times 1^{\circ}$, this could get very large. Consequently, we adapt an approach from compressive sensing to enforce this constraint approximately. Consider a $M_{\mathrm{cs}} \times\left(N_{\mathcal{R}^{\prime}} K\right)$ matrix $\mathbf{R}$, whose rows are direction cosines of random points on the surface of $N_{\mathcal{R}^{\prime}} K$-dimensional unit sphere. This is called a uniform spherical ensemble (Tsaig and Donoho, 2006). The projection of the emission field $\mathbf{F}_{\mathcal{R}^{\prime}}$ on $\mathbf{R}$, i.e., $\mathbf{R F}_{\mathcal{R}^{\prime}}$ compressively samples $\mathbf{F}_{\mathcal{R}^{\prime}}$. $M_{\mathrm{cs}}$ is the number of such projections or compressive samples. Setting this projection to zero during inversion allows us to enforce zero emissions outside $\mathcal{R}$. However, to do so, we add only $M_{\mathrm{cs}}$ constraint equations. The computational savings afforded by imposing the $\mathbf{F}_{\mathcal{R}^{\prime}}=0$ constraint in this manner is investigated in Ray et al. (2013).
The equivalent of Eq. (5) is written as

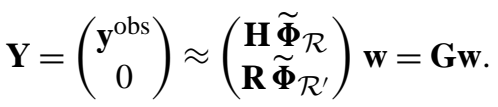

We incorporate the spatial patterns in $\mathbf{X}$ into the estimation procedure by using $\mathbf{w}_{(X)}$ to normalize $\mathbf{w}$. Other, less effective, methods were investigated and discarded in Ray et al. (2013). We rewrite Eq. (8) as

$\mathbf{Y} \approx \mathbf{G} \operatorname{diag}\left(\mathbf{w}_{(X)}\right) \operatorname{diag}\left(\mathbf{w}_{(X)}^{-1}\right) \mathbf{w}=\mathbf{G}^{\prime} \mathbf{w}^{\prime}=\left(\begin{array}{c}\mathbf{H} \widetilde{\boldsymbol{\Phi}}_{\mathcal{R}}^{\prime} \\ \mathbf{R} \widetilde{\boldsymbol{\Phi}}_{\mathcal{R}^{\prime}}^{\prime}\end{array}\right) \mathbf{w}^{\prime}$,

where $\mathbf{w}^{\prime}=\left\{w_{s, i, j} /\left(c w_{(X), s, i, j}\right)\right\},\{s, i, j\} \in W^{(s)}$, is the normalized set of wavelet coefficients, $\widetilde{\boldsymbol{\Phi}}_{\mathcal{R}}^{\prime}=\widetilde{\boldsymbol{\Phi}}_{\mathcal{R}} \operatorname{diag}\left(\mathbf{w}_{(X)}\right)$ and $\widetilde{\boldsymbol{\Phi}}_{\mathcal{R}^{\prime}}^{\prime}=\widetilde{\boldsymbol{\Phi}}_{\mathcal{R}^{\prime}} \operatorname{diag}\left(\mathbf{w}_{(X)}\right)$.

The underdetermined system Eq. (9) is solved using optimization. Given the small number of towers (35) and their location (the towers were sited with biospheric fluxes in mind), it may not be possible to estimate all the elements of $\mathbf{w}^{\prime}$, especially those that contribute to the fine-scale details of $\mathbf{F}_{\mathcal{R}}$. Further, a priori, we do not know the identity of these "unestimatable" wavelet coefficients in $\mathbf{w}^{\prime}$. Consequently, we employ a sparse reconstruction method, based on $\ell_{1}$ minimization, that identifies and estimates the elements of $\mathbf{w}^{\prime}$ that can be constrained by $\mathbf{y}^{\text {obs }}$, while setting the rest to zero. We cast the optimization problem as

$\underset{\mathbf{w}^{\prime} \in \mathbb{R}^{N}}{\operatorname{minimize}}\left\|\mathbf{w}^{\prime}\right\|_{1}, \quad$ subject to $\left\|\mathbf{Y}-\mathbf{G}^{\prime} \mathbf{w}^{\prime}\right\|_{2}^{2}<\epsilon_{2}$.

This is of the same form as Eq. (2) and is solved using stagewise orthogonal matching pursuit (StOMP) (Donoho et al., 2012).

\subsection{Imposing non-negativity on $\mathrm{ffCO}_{2}$ fluxes}

Estimates of $\mathbf{w}^{\prime}$ calculated by StOMP do not necessarily provide non-negative estimates of $\mathbf{F}_{\mathcal{R}}=\widetilde{\boldsymbol{\Phi}}_{\mathcal{R}} \mathbf{w}$. In practice negative $\mathrm{ffCO}_{2}$ emissions occur in only a few grid cells and are usually small in magnitude. We devised an iterative method to impose non-negativity as a post-processing step. We present a summary here; details are in Ray et al. (2013).

We use the StOMP solution to generate $\mathbf{F}=\widetilde{\boldsymbol{\Phi}} \mathbf{w}$, discard $\mathbf{F}_{\mathcal{R}^{\prime}}$ and manipulate the emissions $\mathbf{E}=\left\{E_{i}\right\}, i=1 \ldots N_{\mathcal{R}} K$ in $\mathcal{R}$ directly. We start with a guessed $\mathbf{E}\left(=\left|\mathbf{F}_{\mathcal{R}}\right|\right)$ and at the $m$ th iteration calculate an increment $\Delta \mathbf{E}^{(m-1)}$ to the current iterate $\mathbf{E}^{(m-1)}$

$\mathbf{y}^{\mathrm{obs}}-\mathbf{H} \mathbf{E}^{(m-1)}=\Delta \mathbf{y} \approx \mathbf{H} \Delta \mathbf{E}^{(m-1)}$.

This is an underdetermined problem, and we seek the sparsest set of increments $\Delta \mathbf{E}^{(m-1)}$ using StOMP. The increment is used to calculate a correction $\xi=\left\{\xi_{i}\right\}, i=$ $1 \ldots N_{\mathcal{R}} K,\left|\xi_{i}\right| \leq 1$ and update $\mathbf{E}^{(m-1)}$

$\xi_{i}=\operatorname{sgn}\left(\frac{\Delta E_{i}^{(m-1)}}{E_{i}^{(m-1)}}\right) \max \left(1,\left|\frac{\Delta E_{i}^{(m-1)}}{E_{i}^{(m-1)}}\right|\right)$, 
The iteration is stopped when $\left\|\mathbf{y}^{\mathrm{obs}}-\mathbf{H E}\right\|_{2} /\left\|\mathbf{y}^{\mathrm{obs}}\right\|_{2} \leq \epsilon_{3}$ for a small value of $\epsilon_{3}$.

\section{Numerical tests}

Numerical tests are performed for the domain between the corners $24.5^{\circ} \mathrm{N}, 63.5^{\circ} \mathrm{W}$ and $87.5^{\circ} \mathrm{N}, 126.5^{\circ} \mathrm{W}$. It is discretized by a $2^{M} \times 2^{M}, M=6$ mesh, with 4096 grid cells. Of these, $N_{\mathcal{R}}=816$ cells lie inside $\mathcal{R}$, while the rest, $N_{\mathcal{R}^{\prime}}=$ $4096-816=3280$ lie outside in $\mathcal{R}^{\prime}$. We estimate emissions over $k=1 \ldots K, K=45$, i.e., for $45 \times 8=360$ days (approximately a year). We generate synthetic observations $\mathbf{y}^{\text {obs }}$ using the $\mathrm{ffCO}_{2}$ emissions in Vulcan, which provides them only in $\mathcal{R}$. Hourly Vulcan fluxes are coarsened from $0.1^{\circ}$ resolution to $1^{\circ}$, and averaged to 8-day periods. These fluxes are multiplied by $\mathbf{H}$ to obtain $\mathrm{ffCO}_{2}$ concentrations at the $N_{\mathrm{s}}=$ 35 measurement towers. Observations are available every $3 \mathrm{~h}$ and span a full year, i.e., we collect $K_{\mathrm{s}}=24 / 3 \times 360=2880$ observations per tower. A measurement error $\epsilon \sim N\left(0, \sigma^{2}\right)$ is added to the concentrations to obtain $\mathbf{y}^{\text {obs }}$, as used in Eq. (8). The same $\sigma$ is used for all towers and is set to a very low value of $0.1 \mathrm{ppmv}$. Although such a value is unrealistically small for real-data inversions, it is used here to isolate the impact of the proposed parameterization and inversion approach.

We solve Eq. (10) and enforce non-negativity on $\mathbf{F}_{\mathcal{R}}$ to obtain $\mathbf{E}$. The coefficients $\mathbf{w}_{(\mathbf{X})}$ used in Eq. (9) are obtained from a wavelet decomposition of $\mathbf{f}_{\mathrm{pr}}$ based on nightlights (Sect. 3). The constant $c$ in Eq. (4) is obtained by using fluxes from the Emission Database for Global Atmospheric Research (EDGAR, http://edgar.jrc.ec.europa.eu; Olivier et al., 2005) for 2005, i.e., instead of using emissions from Vulcan to calculate $\overline{\mathbf{f}_{\mathrm{V}}}$, we use EDGAR. EDGAR emissions aggregated over $\mathcal{R}$ are $7.1 \%$ higher than $\overline{\mathbf{f}_{\mathrm{V}}}$, resulting in a correspondingly higher $c$. The RMSE between the two is $0.035 \mu$ moles $\mathrm{m}^{-2} \mathrm{~s}^{-1}$ of $\mathrm{C}$ and the Pearson correlation coefficient is 0.726 . Also, since $c$ is an aggregate total over $\mathcal{R}$, it reflects the I.E.A country total. The following parameters are used in the inversion process (Sect. 4.2): $\epsilon_{2}=10^{-5}, \epsilon_{3}=$ $5.0 \times 10^{-4}, M_{\mathrm{cs}}=13500$, i.e., 300 compressive samples for each 8-day period. The numerical values of $\epsilon_{2}$ and $\epsilon_{3}$ were set by reducing them till the solution $\mathbf{w}^{\prime}$ became insensitive to them. The setting for $M_{\mathrm{cs}}$ is more involved and is described in Ray et al. (2013). The initial guess for $\mathbf{w}^{\prime}$ in Eq. (10) is zero.

In Fig. 7 we plot the cumulative distribution function $(\mathrm{CDF})$ of $\mathrm{ffCO}_{2}$ emissions before and after the enforcement of non-negativity. We see the existence of a few grid cells with negative fluxes, but their magnitudes are not very large. Thus the sparse reconstruction scheme provides a good starting guess for the imposition of non-negativity via the iterative method described in Sect. 4. In Fig. 8, we plot the true and reconstructed emissions for the 33rd 8-day period $(k=33)$. We also plot the estimation error $\left(E_{k}-\mathbf{f}_{\mathrm{V}, k}\right)$, averaged over

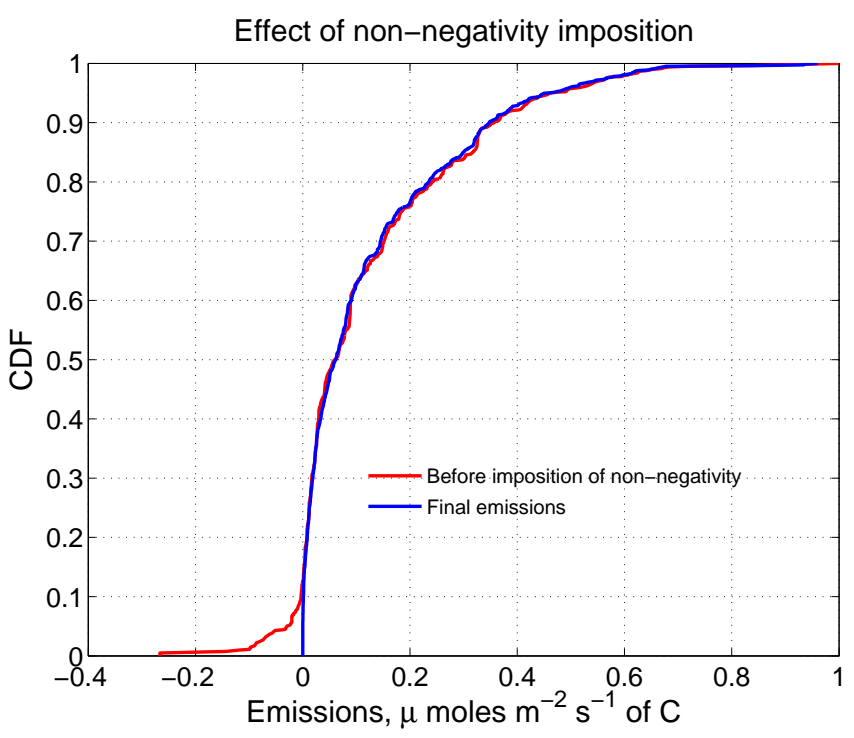

Figure 7. CDF of emissions in $\mathcal{R}$, before and after the imposition of non-negativity, as described in Sect. 4 . We see that the CDF of the emissions without non-negativity imposed contains a few grid cells with negative fluxes; further, the magnitude of the negative emissions is small. Thus the spatial parameterization, with sparse reconstruction, provides a good approximation of the final, non-negative emissions.

the 32-day period $33 \leq k \leq 36$. We see that the reconstruction in the NE quadrant is qualitatively similar to the true emissions. In contrast, the reconstruction on the west coast contains significant inaccuracies. For example, we see that the Los Angeles-San Diego region (southwest quadrant) is estimated incorrectly. The estimated emissions in the center of the country (Continental Divide and Great Plains, in the western quadrants) show similar errors, as well as far more structure than the true $\mathrm{ffCO}_{2}$ emissions. The region around the Gulf of Mexico is also not well estimated. The quality of the reconstruction in the various regions correlate with the density of observations towers, though the wind fields also play an important part. In the regions where the observations are not very informative, the impact of normalization by $\mathbf{f}_{\mathrm{pr}}$ is clear as some of its structure is retained in the estimated emissions. These errors are almost entirely at fine spatial scales.

In Fig. 9 (top) we plot a time-series of errors defined as a percentage of total, country-level Vulcan emissions. Percent errors in reconstructed emissions and $\mathbf{f}_{\mathrm{pr}}$ are calculated using Eq. (13). 

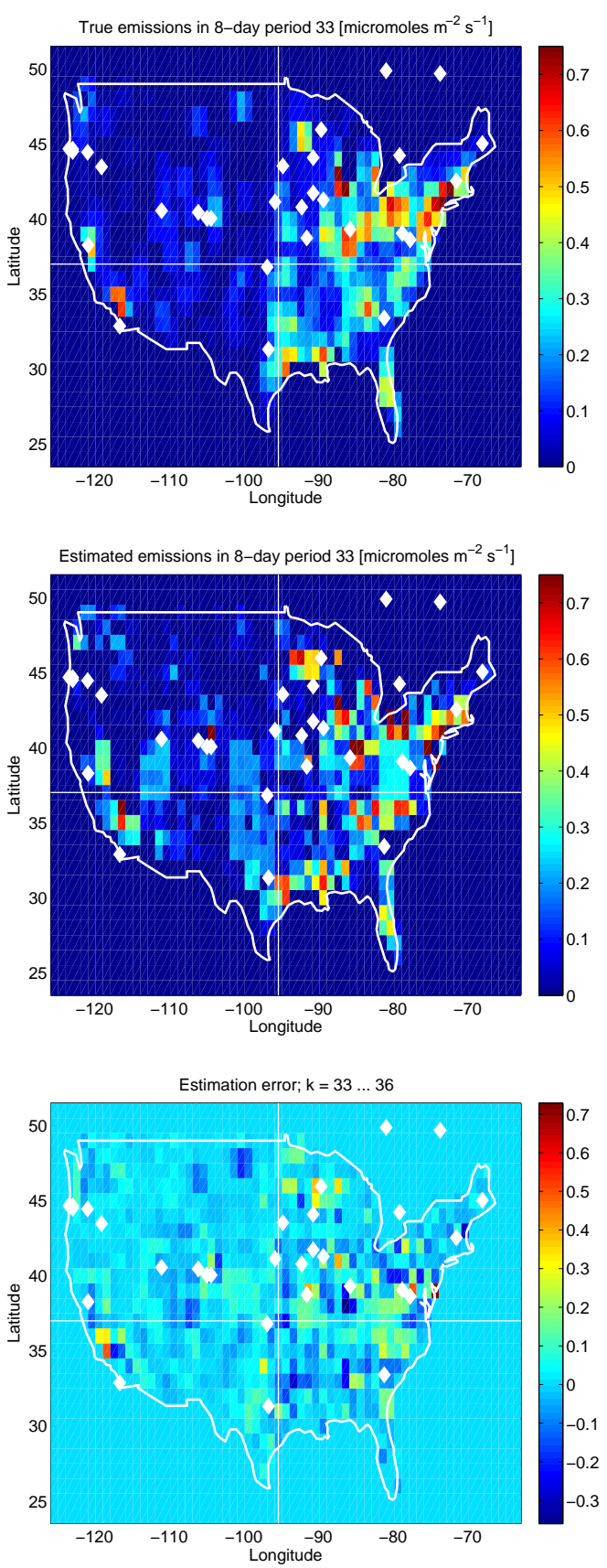

Figure 8. Reconstruction of the $\mathrm{ffCO}_{2}$ emissions from the 35 towers (plotted as diamonds). The true emissions are on top and the reconstructions in the middle. The figures represent emissions for $k=33$ (end of August). At the bottom, we plot the estimation error, $\left(E_{k}-\mathbf{f}_{V, k}\right)$, averaged over $33 \leq k \leq 36$. We see that the large-scale structure of the emissions have been captured. The west coast of the US has few towers near heavily populated regions and thus is not very well estimated. On the other hand, due to the higher density of towers in the northeast, the true and estimated emissions are qualitatively similar and estimation error are low. Emissions have units of $\mu \mathrm{mol} \mathrm{m}{ }^{-2} \mathrm{~s}^{-1}$ of $\mathrm{C}\left(\operatorname{not} \mathrm{CO}_{2}\right)$.

$$
\begin{aligned}
& \text { Error }_{k}(\%)=\frac{100}{K} \sum_{k=1}^{K} \frac{E_{k}-E_{\mathrm{V}, k}}{E_{\mathrm{V}, k}}, \\
& \text { where } E_{k}=\int_{\mathcal{R}} \mathbf{E}_{k} \mathrm{~d} A \\
& \text { and } E_{\mathrm{V}, k}=\int_{\mathcal{R}} \mathbf{f}_{\mathrm{V}, k} \mathrm{~d} A, \\
& \text { Error } \mathrm{pr}, k(\%)=\frac{100}{K} \sum_{k=1}^{K} \frac{E_{\mathrm{pr}}-E_{\mathrm{V}, k}}{E_{\mathrm{V}, k}}, \\
& \text { where } E_{\mathrm{pr}}=\int_{\mathcal{R}} \mathbf{f}_{\mathrm{pr}} \mathrm{d} A .
\end{aligned}
$$

Here, $\mathbf{f}_{\mathrm{V}, k}$ are Vulcan emissions averaged over the $k$ th 8 day period and $\mathbf{E}_{k}$ are the non-negativity enforced emission estimates in the same time period. A positive error denotes an overestimation by the inverse problem. We see $25 \%$ errors in $\mathbf{f}_{\mathrm{pr}}$. The large error is a consequence not only of the disagreement between EDGAR (from 2005) and Vulcan (from 2002), but also the manner in which they account for emissions. As can be seen, assimilation of $\mathbf{y}^{\text {obs }}$ reduces the error significantly vis-à-vis $\mathbf{f}_{\mathrm{pr}}$. The least accurate reconstructions are during spring $(k=10-15)$. In order to check the accuracy of the spatial distribution of $\mathbf{E}_{k}$, we calculate the Pearson correlations $\rho\left(\mathbf{E}_{k}, \mathbf{f}_{\mathrm{V}, k}\right)$ and $\rho\left(\mathbf{f}_{\mathrm{pr}}, \mathbf{f}_{\mathrm{V}, k}\right)$. We see that data assimilation results in a clear increase in the correlation. When the emissions are aggregated/averaged over 32-day periods, the correlation increases to about 0.85 , whereas the "prior" correlation was around 0.7 . Thus the $\mathrm{ffCO}_{2}$ emissions obtained using a nightlight proxy are substantially improved by the incorporation of $\mathbf{y}^{\text {obs }}$. Only about half the wavelet coefficients could be estimated; the rest were set to zero by the sparse reconstruction technique (Ray et al., 2013).

We next investigate the effect of using BUA maps, instead of nightlights, as the proxy. Changing the proxy results in a different set of wavelets being chosen (nightlights resulted in a $W^{(s)}$ of 1031 wavelets; the corresponding number for BUA was 1049); further, one was not a strict subset of the other. It also results in a different normalization in Eq. (9). The inversion was performed in a manner identical to that adopted for the nightlight proxy. In Fig. 9 (top) we see that the $\mathrm{ffCO}_{2}$ emissions developed using nightlights and BUA as proxies are similar, as measured by reconstruction error (Eq. 13), though the BUA reconstruction error tends to be slightly smaller. The aggregated error between the true and "prior" fluxes remains unchanged (nightlights vs. BUA) since it just reflects the difference between EDGAR (in 2005) and Vulcan (in 2002) inventories. In Fig. 9 (bottom) we plot the spatial correlation between the true, reconstructed and "prior" fluxes. The correlation between true and reconstructed emissions (from BUA) tends to be worse than the nightlight reconstruction. The correlation of $\mathbf{f}_{\mathrm{pr}}$ with true 

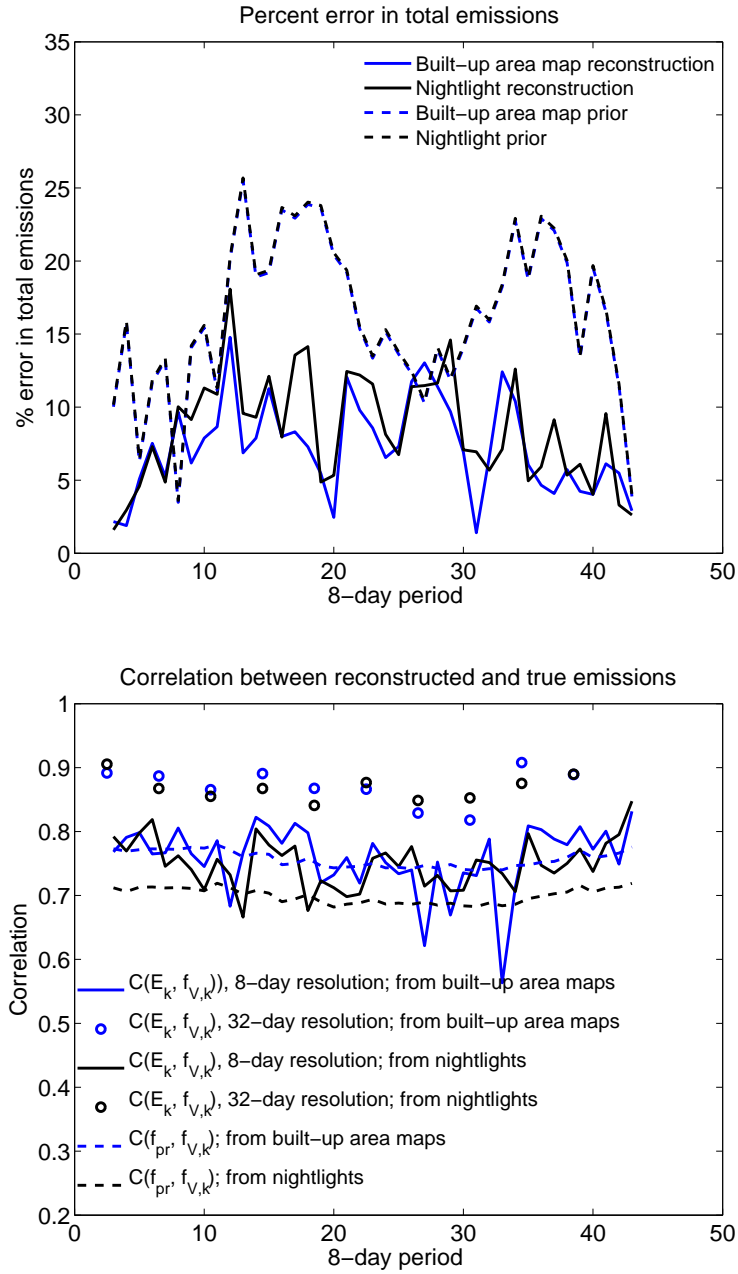

Figure 9. Comparison of reconstruction error and correlations. Top: we plot the error between the reconstructed and true (Vulcan) emissions in black (using nightlights as priors) and in blue (using BUA priors). We plot the error between $\mathbf{f}_{\mathrm{pr}}$ and Vulcan emissions using dashed lines - black for nightlights and blue for BUA. We see that assimilation of $\mathbf{y}^{\text {obs }}$ leads to significantly improved accuracy vis-àvis $\mathbf{f}_{\mathrm{pr}}$. Bottom: we plot the accuracy of the spatial distribution of the reconstructed emissions. The Pearson correlations $\rho\left(\mathbf{E}_{k}, \mathbf{f}_{\mathrm{V}, k}\right)$ and $\rho\left(\mathbf{f}_{\mathrm{pr}}, \mathbf{f}_{\mathrm{V}, k}\right)$ show that incorporating $\mathbf{y}^{\text {obs }}$ marginally improves the spatial agreement of estimated emissions vs. the true one when using nightlights, though the results are less clear for BUA priors. If the emissions are averaged over 32-day periods, rather than 8-day periods, the correlation with true (Vulcan) emissions rises to around 0.85 , irrespective of the prior used.

emissions from Vulcan are different for nightlights and BUA reflecting the distinct spatial difference between them as seen in Fig. 6. This results in the difference between the two dashed lines. Averaging over 32-day intervals improves the correlation and makes them almost indistinguishable from those obtained using nightlights.
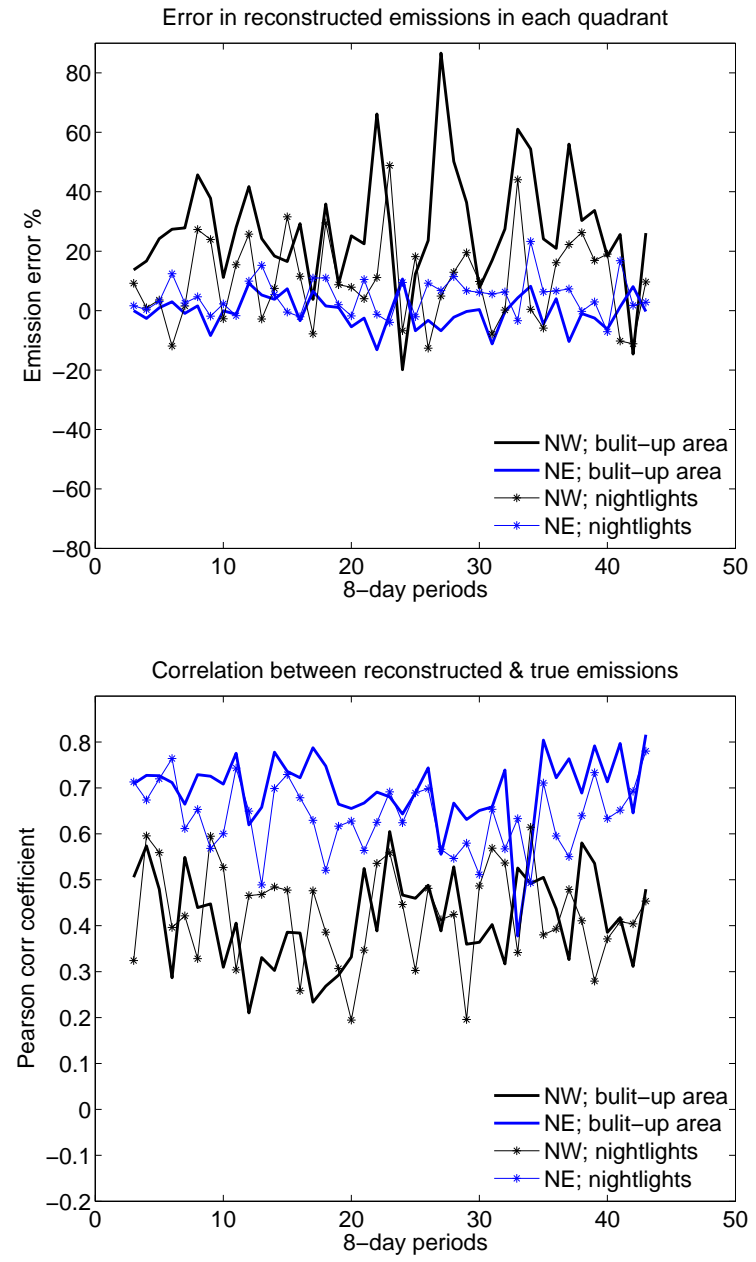

Figure 10. Top: emission reconstruction error in the NE (blue) and NW (black) quadrants, when performed with BUA (line) and nightlights (symbols) as proxies. We see that the NW quadrant is very badly constrained and the BUA-based estimates have very large errors. The errors in the NE quadrant are far smaller and very similar when generated using the competing proxies. Bottom: the comparison of correlations between true and reconstructed emissions shows similar trends.

In Fig. 10 we investigate the differences between the nightlight- and BUA-based reconstructions at the quadrant level. We see in Fig. 10 (top) that the difference between nightlight- and BUA-based reconstruction errors in the NE quadrant are smaller than those for the NW quadrant. Thus, while the $\mathbf{f}_{\text {pr }}$ from nightlights and BUA are quite different (see the last row of Fig. 6), the estimated emissions are well informed by $\mathbf{y}^{\mathrm{obs}}$ in the NE quadrant and the impact of the proxies is small. This is not the case for the NW quadrant, where the reconstruction based on BUA is clearly much worse than the nightlight-based reconstruction. This is not surprising given the paucity of towers there (see Fig. 8), which increases the impact of $\mathbf{f}_{\mathrm{pr}}$. In Fig. 10 (bottom) we plot the correlation of the reconstructed and true emissions 

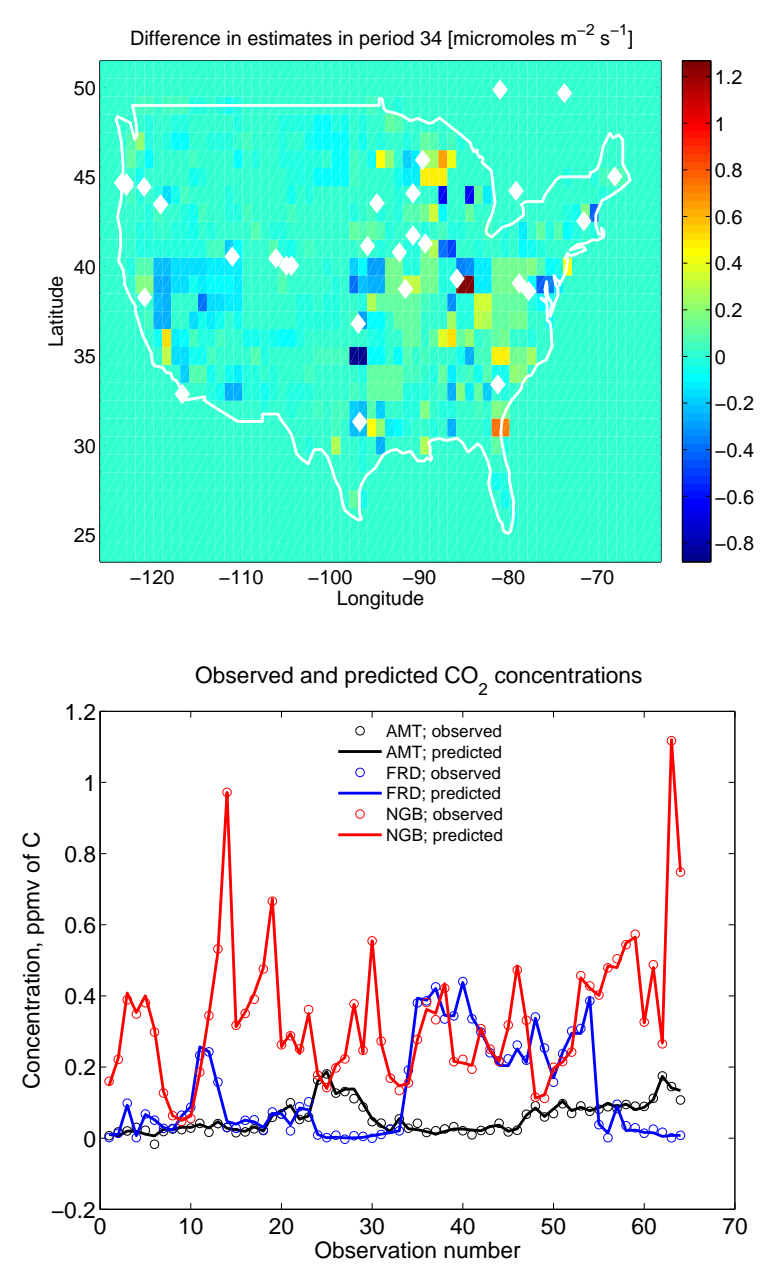

Figure 11. Top: comparison of emission estimates developed using $\mathbf{f}_{\text {pr }}$ constructed from nightlight radiances and BUA maps. We plot the difference between the two estimates. We see that differences are not localized in any one area. Bottom: prediction of $\mathrm{ffCO}_{2}$ concentrations at three measurement locations, using the true (Vulcan; plotted with symbols) and reconstructed emissions (blue lines) over an 8-day period (Period no. 31). Observations occur every $3 \mathrm{~h}$. We see that the concentrations are accurately reproduced by the estimated emissions.

in the NE and NW quadrants. We see that there is little to choose between the correlations generated using nightlightvs. the BUA-based emission estimates. Again, due to the larger density of towers in the NE, the correlations are higher there. Thus, while Fig. 6 (middle row) showed that BUA had a slightly better correlation with true (Vulcan) emissions, its larger errors, as seen in Fig. 6 (bottom row) lead to a less accurate reconstruction. This result is also a testament to the inadequacy of $\mathbf{y}^{\text {obs }}$ over the whole country for constraining $\mathrm{ffCO}_{2}$ emissions; had there been sufficient data to inform $\mathbf{E}$, the impact of $\mathbf{f}_{\mathrm{pr}}$ would have been minimal.

Next, in Fig. 11 (top) we compare the estimated emissions for the 34th 8-day period developed from the two competing prior models. We plot the difference between the two estimates; it shows differences spread over a large area, though their magnitudes are not very big. Thus the "prior" model has a measurable impact on the spatial distribution of the emissions. In Fig. 11 (bottom) we plot $\mathbf{y}$ predicted by the reconstructed emissions (from nightlights as priors) at 3 towers. The towers were chosen to represent the range of the $\mathrm{ffCO}_{2}$ signal strengths encountered in our test cases. We see that the $\mathrm{ffCO}_{2}$ concentrations are well reproduced by the estimated emissions. Further, note that the measurement noise $(\sigma=0.1 \mathrm{ppmv})$ is relatively large compared to some of the observations. Thus, the lack of fidelity at the smaller scales (seen in Fig. 8) does not substantially impact the measurements. This is due to the weak strengths of the erroneous emission sources (while a few may be intense, they are present only over a small area) and their distance from the towers.

\section{Discussion}

The numerical results in Sect. 5 show that the MsRF and sparse reconstruction techniques can solve the inverse problem as formulated in Sect. 4, conditioned on limited measurements of $\mathrm{ffCO}_{2}$ concentrations. The solution reproduces large-scale spatial patterns of the true flux field, and some of the finer ones. The rough spatial nature of the emission field is preserved in the estimates. Furthermore, the method is insensitive to underreporting of $\mathrm{ffCO}_{2}$ emissions by countries which are used to construct inventories such as EDGAR. Inventories are used only to calculate $c$ in Eq. (4) which appears as a normalization constant in Eq. (9). The accuracy of the estimates (the constraint $\left\|\mathbf{Y}-\mathbf{G}^{\prime} \mathbf{w}^{\prime}\right\|_{2}^{2}<\epsilon_{2}$ in Eq. (10)) is unaffected by the value of $c$. The chief source of errors in the estimates is the paucity of observations sensitive to fossilfuel-emitting regions. Regions with low tower density, e.g., the western quadrants in Fig. 8, have large errors due to the faint $\mathrm{ffCO}_{2}$ signal at existing observational sites. One limitation of the deterministic estimation method presented here is that it does not provide any measure of the uncertainty in the estimates. The numerical parameters $\epsilon_{2}, \epsilon_{3}$ and $M_{\mathrm{cs}}$ are not significant sources of uncertainty since they were set at values where the solution of the inverse problem became insensitive to them.

Given our focus on the algorithmic issues in the estimation of $\mathrm{ffCO}_{2}$ emissions under realistic conditions, the inverse problem that we constructed is idealized and embodies a number of simplifications. We have used a sensor network (that existed in 2008) that was sited with an eye towards estimating biospheric $\mathrm{CO}_{2}$ fluxes. This network is therefore not optimized for constraining $\mathrm{ffCO}_{2}$ emission sources, leading to a faint $\mathrm{ffCO}_{2}$ signal $(<2 \mathrm{ppmv})$. This made the use of a small model-data mismatch error necessary for the synthetic-data experiments presented here $(\sigma=$ $0.1 \mathrm{ppmv}$ ), a value that would not be realistic for inversions using real data. The experiments conducted here also assume 
the availability of observations that isolate the $\mathrm{ffCO}_{2}$ signal, which would either require observations of a $\mathrm{ffCO}_{2} \mathrm{~ns}-$ specific tracer, or the pre-subtraction of the influence of biospheric fluxes from observations. Some of the other simplifications used in the setup, on the other hand, are common to synthetic-data inversion experiments focusing on biospheric fluxes and reported elsewhere, e.g., Gourdji et al. (2010). For example, we have ignored emissions outside $\mathcal{R}$; in a realistic ffCO $_{2}$ estimation problem, emissions outside $\mathcal{R}$ would have to be modeled as boundary conditions to the examined domain, as is done for regional biospheric inversion studies. Furthermore, we have also assumed a constant data-model mismatch $(\sigma)$ across all sensors, and site- and seasonally varying model-data mismatch statistics would be required when real data are used.

The use of proxies to construct the MsRF for $\mathrm{ffCO}_{2}$ emissions can be a source of estimation errors and consequently, in Sects. 3 and 5, we investigated nightlights and BUA maps to explore the impact of using such proxies for sub-selecting the wavelets to be used in the inversion. Errors in the proxies themselves (i.e. inaccuracies in the nightlights and BUA data themselves) are unlikely to be a large source of estimation errors in the inversion, as these proxies are used only to select wavelets, whereas the wavelet coefficients are obtained in the inversion step. Rather, inversion errors can stem from the fact that nightlights correlate with energy consumption and not energy production. This can lead to two types of errors: (1) when a fine-scale wavelet covering a region with a strong $\mathrm{ffCO}_{2}$ source and little human habitation is omitted from the MsRF and (2) when we choose a "superfluous" fine-scale wavelet in a region with much human activity and little emission. An example of the first type of error is large powerplants, which are usually sited far from densely populated areas. In such a case, the point-source is modeled by the coarse-scale wavelet that covers the area in question, leading to a "smeared" reconstruction. Such large point-sources of $\mathrm{ffCO}_{2}$ emissions could instead be obtained from databases such as CARMA and incorporated directly into the inversion. Alternatively, one could augment the wavelets in the MsRF with those chosen using a second proxy, e.g., thermal images, where large emitters of heat can be easily detected. The second type of error, that of the "superfluous" wavelet, is rectified when it is simply removed by the sparse reconstruction scheme in the inversion step. An exception can occur if the superfluous wavelet contains a measurement tower in its support and is far from all other towers. Since measurement towers are very sensitive to fluxes in their vicinity (Gerbig et al., 2009), it could lead to the estimation of a spurious emission source.

$\mathrm{ffCO}_{2}$ emissions, averaged over 8-day intervals and predicated on 3-hourly measurements, were estimated as independent variables, i.e., without imposing a temporal correlation or modeling their temporal evolution in any way. The reason is as follows. Estimation of $\mathrm{ffCO}_{2}$ emissions over a 8-day interval requires the calculation of 1031 wavelet coefficients (when using the nightlights-derived MsRF) from $35 \times 8 \times 8=2240$ measurements. This is not an underdetermined problem, even though a sparse reconstruction method was required to remove fine-scale structures (wavelets) in the emission field that did not affect the measurements. We were able to constrain the coefficients of the remaining wavelets without imposing a temporal correlation structure. Such correlations could be used if $\mathrm{ffCO}_{2}$ fluxes were to be estimated at finer temporal resolution.

The spatial parameterization and the sparse reconstruction method can also be used in observation system simulation experiments (OSSEs) to inform the design of measurement networks targeted for $\mathrm{ffCO}_{2}$ emissions. The approach can be used to decide locations of towers, the frequency at which $\mathrm{ffCO}_{2}$ measurements are to be made, and the fidelity required in measurements and the transport model. The trade-offs and costs of various $\mathrm{ffCO}_{2}$ measurement technologies can also be studied in such a setting. In addition, OSSEs can reveal the importance of a more accurate MsRF, e.g., one augmented using thermal imagery, vs. the errors introduced in the estimates due to limited measurements.

\section{Conclusions}

We have devised a multiresolution parametrization (also known as a multiscale random field or MsRF model) for modeling $\mathrm{ffCO}_{2}$ emissions at $1^{\circ}$ resolution. The MsRF models emissions in the lower 48 states of the US and is designed for use in atmospheric inversions. The parameterization employs Haar wavelets which provide a sparser representation than other smoother wavelets with wider support. This is the first "abstract" parameterization, i.e., a RF model for spatially resolved $\mathrm{ffCO}_{2}$ emissions.

The dimensionality of the MsRF was reduced by judiciously selecting its component Haar wavelets using proxies of human activity, and therefore indicative of $\mathrm{ffCO}_{2}$ emissions. We developed two MsRFs based on images of lights at night and maps of built-up areas. The former had a slightly lower dimensionality but was not a strict subset of the latter. The MsRF models were also used to develop two approximate emission models that differed in their fine spatial details.

The MsRF model was tested in a synthetic-data inversion. Time-dependent $\mathrm{ffCO}_{2}$ emissions, averaged over 8-day periods, were estimated for a 360-day period from measurements of $\mathrm{ffCO}_{2}$ concentrations at 35 towers. These observations were sufficient only for estimating about half the wavelets retained in the MsRF model. We used a sparse reconstruction technique, namely Stagewise Matching Orthogonal Pursuit (StOMP), to identify and estimate wavelet coefficients in MsRF that could be informed by the available data. The StOMP estimates were not necessarily non-negative (as $\mathrm{ffCO}_{2}$ emissions are required to be) and we devised an iterative, post-processing procedure to impose non-negativity. 
Furthermore, the MsRF actually models emissions in a rectangular region and constraints had to be imposed during the inversion to ensure that emissions were restricted to the lower 48 states. To our knowledge, this is the first instance of using a sparse reconstruction method in atmospheric $\mathrm{CO}_{2}$ inversions. The algorithmic details of the inversion procedure are in Ray et al. (2013). This is also the first use of an MsRF model in $\mathrm{ffCO}_{2}$ emission estimation.

The inversions using the competing MsRF models (nightlights- vs. built-up-area-based) resulted in emission estimates that differed in their fine-scale details. Country-level estimates of emissions, and their correlation with the true emissions, differed little when developed using either of the two MsRFs. Further, emissions obtained by dividing the US into quadrants showed large errors vis-à-vis true emissions. This is a testament to the fact that the current observations network was designed to focus on biospheric rather than fossil-fuel $\mathrm{CO}_{2}$ fluxes.

The spatial parameterization and its use in reconstructing an emission field was demonstrated in an idealized setting. A number of adaptions are required before it can be used in a realistic scenario to infer $\mathrm{ffCO}_{2}$ emissions. These include aspects that are standard to any regional inversion, such as accounting for fluxes outside the modeled domain via boundary conditions, and accommodating errors incurred by the transport model. Results using even our idealized setup show that constraining fossil-fuel emissions at regional to national scales would require a measurement network better targeted to that goal. In addition to the prototypical application presented here, our approach can also be used in OSSEs for designing such a measurement network - determining optimal locations of towers, intervals between measurements, and the fidelity required in the transport model and the measurements themselves.

Our inversion formulation suffers from two drawbacks. One is the need for observations of $\mathrm{ffCO}_{2}$ concentrations at the measurement towers, which could be obtained either by making use of co-emitted tracers or by pre-subtracting the influence of biospheric fluxes from observations. Neither of these, however, is easily achievable given current observational constraints and uncertainties associated with biospheric fluxes. The second drawback is the deterministic nature of the reconstruction, which does not provide error bounds on the estimates of the MsRF parameters (the wavelet coefficients).

In conjunction with this paper, we are also providing the MATLAB ${ }^{\circledR}$ code required to (1) reduce the dimensionality of the MsRF using nightlights and (2) perform the inversion using synthetic observations at our website (Ray, 2013). The website also contains links to the (free) MATLAB toolkits that our code depends on, along with a user's manual.

The Supplement related to this article is available online at doi:10.5194/gmd-7-1901-2014-supplement.
Acknowledgements. Thanks are due to Sharon Gourdji, Kim Mueller and Abhishek Chatterjee for fruitful discussions regarding algorithms, the challenges of working with field observations as well as some of the data used in this work. This work was supported by Sandia National Laboratories' LDRD (Laboratory Directed Research and Development) funds, sponsored by the Geosciences Investment Area. Sandia National Laboratories is a multi-program laboratory managed and operated by Sandia Corporation, a wholly owned subsidiary of Lockheed Martin Corporation, for the U.S. Department of Energy's National Nuclear Security Administration under contract DE-AC04-94AL85000.

Edited by: A. Stenke

\section{References}

Andres, R. J., Boden, T. A., Bréon, F.-M., Ciais, P., Davis, S., Erickson, D., Gregg, J. S., Jacobson, A., Marland, G., Miller, J., Oda, T., Olivier, J. G. J., Raupach, M. R., Rayner, P., and Treanton, K.: A synthesis of carbon dioxide emissions from fossil-fuel combustion, Biogeosciences, 9, 1845-1871, doi:10.5194/bg-91845-2012, 2012.

Auger, L. and Tangborn, A. V.: A wavelet-based reduced rank Kalman filter for assimilation of stratospheric chemical tracer observations, Mon. Weather Rev., 132, 1220-1237, 2002.

Brioude, J., Petron, G., Frost, G. J., Ahmadov, R., Angevine, W. M., Hsie, E.-Y., Kim, S.-W., Lee, S.-H., McKeen, S. A., Trainer, M., Fehsenfeld, F. C., Holloway, J. S., Peischl, J., Ryerson, T. B., and Gurney, K. R.: A new inversion method to calculate emission inventories without a prior at mesoscale: application to the anthropogenic $\mathrm{CO}_{2}$ emissions from Houston, Texas, J. Geophys. Res., 117, D05312, doi:10.1029/2011JD016918, 2012.

Candes, E. and Tao, T.: Near optimal signal recovery from random projections: universal encoding strategies?, IEEE T. Inform. Theory, 52, 5406-5425, 2006.

Candes, E. and Wakin, M.: An introduction to compressive sampling, IEEE Signal Proc. Mag., 25, 21-30, 2008.

Candes, E., Romberg, J., and Tao, T.: Robust uncertainty principles: exact signal reconstruction from highly incomplete frequency information, IEEE T. Inform. Theory, 52, 489-509, 2006.

Chan, T. F. and Shen, J. J.: Image Processing and Analysis - Variational, PDE, Wavelet, and Stochastic Methods, Society of Applied Mathematics, 2005.

Chatterjee, A., Michalak, A. M., Mueller, K. L., Anderson, J. L., and Yadav, V.: Towards reliable ensemble Kalman filter estimates, J. Geophys. Res.-Atmos., 117, D22306, doi:10.1029/2012JD018176, 2012.

Chen, S., Donoho, D., and Saunders, M.: Atomic decomposition by basis pursuit, SIAM J. Sci. Comput., 20, 33-61, 1998.

Ciais, P., Rayner, P., Chevallier, F., Bousquet, P., Logan, M., Peylin, P., and Ramonet, M.: Atmospheric inversions for estimating $\mathrm{CO}_{2}$ fluxes: methods and perspectives, Climate Change, 103, 69-92, 2010.

Cinzano, P., Falchi, F., Elvidge, C. D., and Baugh, K. E.: The artificial night sky brightness mapped from DMSP satellite Operational Linescan System measurements, Mon. Not. R. Astron. Soc., 318, 641-657, 2000.

Cressie, N. A. C.: Statistics for spatial data, Wiley-Interscience, 1993. 
Doll, C. N. H., Muller, J.-P., and Elvidge, C. D.: Night-time imagery as a tool for global mapping of socioeconomic parameters and greenhouse gas emissions, Ambio, 29, 157-162, 2000.

Donoho, D. L.: Denoising by soft thresholding, IEEE T. Inform. Theory, 41, 613-627, 1995.

Donoho, D.: Compressed sensing, IEEE T. Inform. Theory, 52, 1289-1306, 2006.

Donoho, D. L., Tsaig, Y., Drori, I., and Starck, J.-L.: Sparse solution of underdetermined linear equations by stagewise orthogonal matching pursuit, IEEE T. Inform. Theory, 58, 1094-1121, 2012.

Duarte, M. F., Wakin, M. B., and Baraniuk, R. G.: Wavelet-domain compressive signal reconstruction using a hidden Markov tree mode, in: Proc. IEEE International Conference on Acoustics, Speech, and Signal Processing (ICASSP), Las Vegas, NV, 2008.

Friedlingstein, P., Cox, P., Betts, R., Bopp, L., von Bloh, W., Brovkin, V., Cadule, P., Doney, S., Eby, M., Rung, I., Bala, G., John, J., Jones, C., Joos, F., Kato, T., Kawamiya, M., Knor, W., Lindsay, K., matthews, H. D., Raddatz, T., Rayner, P., Reick, C., Roeckner, E., Schitzler, K.-G., Schur, R., Strassmann, K., Weaver, A. J., Yoshikawa, C., and Zeng, N.: Climate-Carbon cycle feedback analysis: results from the C4MIP model intercomparison, J. Climate, 19, 3337-3353, 2006.

Gerbig, C., Dolman, A. J., and Heimann, M.: On observational and modelling strategies targeted at regional carbon exchange over continents, Biogeosciences, 6, 1949-1959, doi:10.5194/bg6-1949-2009, 2009.

Gholami, A. and Siahkoohi, H. R.: Regularization of linear and nonlinear geophysical ill-posed problems with joint sparsity priors, Geophys. J. Int., 180, 871-882, 2010.

Goldstein, T. and Osher, S.: The Split Bregman Method for L1Regularized Problems, SIAM J. Imaging Sciences, 2, 323-343, 2009.

Gourdji, S. M., Hirsch, A. I., Mueller, K. L., Yadav, V., Andrews, A. E., and Michalak, A. M.: Regional-scale geostatistical inverse modeling of North American $\mathrm{CO}_{2}$ fluxes: a synthetic data study, Atmos. Chem. Phys., 10, 6151-6167, doi:10.5194/acp-10-61512010, 2010.

Gourdji, S. M., Mueller, K. L., Yadav, V., Huntzinger, D. N., Andrews, A. E., Trudeau, M., Petron, G., Nehrkorn, T., Eluszkiewicz, J., Henderson, J., Wen, D., Lin, J., Fischer, M., Sweeney, C., and Michalak, A. M.: North American $\mathrm{CO}_{2}$ exchange: inter-comparison of modeled estimates with results from a fine-scale atmospheric inversion, Biogeosciences, 9, 457-475, doi:10.5194/bg-9-457-2012, 2012.

Gregg, J. S., Andres, R. J., and Marland, G.: China: emission pattern of the world leader in $\mathrm{CO}_{2}$ emissions from fossil fuel consumption and cement production, Geophys. Res. Lett., 35, L08806, doi:10.1029/2007GL032887, 2008.

Gurney, K. R., Mendoza, D. L., Zhou, Y., Fischer, M. L., Miller, C. C., Geethakumar, S., and de la Rue de Can, S.: High resolution fossil fuel combustion $\mathrm{CO}_{2}$ emission fluxes in the United States, Environ. Sci. Technol., 43, 5535-5541, 2009.

Gurney, K. R., Razlivanov, I., Song, Y., Zhou, Y., Benes, B., and Abdul-Massih, M.: Quantification of fossil fuel $\mathrm{CO}_{2}$ emissions on the building/street scale for a large city, Environ. Sci. Technol., 46, 12194-12202, 2012.
He, L. and Carin, L.: Exploiting structure in wavelet-based Bayesian compressive sensing, IEEE T. Signal Proc., 57, 34883497, 2009.

Jafarpour, B.: Wavelet reconstruction of geologic facies from nonlinear dynamic flow measurements, IEEE T. Geosci. Remote, 49, 1520-1535, 2011.

Jafarpour, B.: Sparsity-promoting solution of subsurface flow model calibration inverse problems, in: Advances in Hydrogeology, edited by: Mishra, P. K. and Kuhlman, K. L., Springer, 2013.

Li, L. and Jafarpour, B.: A sparse Bayesian framework for conditioning uncertain geologic models to nonlinear flow measurements, Adv. Water Resour., 33, 1024-1042, 2010.

Lin, J. C., Gerbig, C., Wofsy, S. C., Andrews, A. E., Daube, B. C., Davis, K. J., and Grainger, C. A.: A near-field tool for simulating the upstream influence of atmospheric observations: the Stochastic Time-Inverted Lagrangian Transport (STILT) model, J. Geophys. Res., 108, 4493, doi:10.1029/2002JD003161, 2003.

Loris, I., Nolet, G., Daubechies, I., and Dahlen, F. A.: Tomographic inversion using $\ell_{1}$-norm regularization of wavelet coefficients, Geophys. J. Int., 170, 359-370, 2007.

Mallat, S. and Zhang, Z.: Matching pursuit with time-frequency dictionaries, IEEE T. Signal Proc., 41, 3397-3415, 1993.

McKain, K., Wofsy, S., Nehrkorn, T., Eluszkiewicz, J., Ehrlinger, J. R., and Stephens, B. B.: Assessment of groundbased atmospheric observations for verification of greenhouse gas emissions from an urban region, Proc. Natl. Acad. Sci. USA, 109, 8423-8428, 2012.

Nassar, R., Jones, D. B. A., Kulawik, S. S., Worden, J. R., Bowman, K. W., Andres, R. J., Suntharalingam, P., Chen, J. M., Brenninkmeijer, C. A. M., Schuck, T. J., Conway, T. J., and Worthy, D. E.: Inverse modeling of $\mathrm{CO}_{2}$ sources and sinks using satellite observations of $\mathrm{CO}_{2}$ from TES and surface flask measurements, Atmos. Chem. Phys., 11, 6029-6047, doi:10.5194/acp-11-60292011, 2011.

Oda, T. and Maksyutov, S.: A very high-resolution $(1 \mathrm{~km} \times 1 \mathrm{~km})$ global fossil fuel $\mathrm{CO}_{2}$ emission inventory derived using a point source database and satellite observations of nighttime lights, Atmos. Chem. Phys., 11, 543-556, doi:10.5194/acp-11-543-2011, 2011.

Olivier, J. G. J., Aardenne, J. A. V., Dentener, F. J., Pagliari, V., Ganzeveld, L. N., and Peters, J. A. H. W.: Recent trends in global greenhouse gas emissions: regional trends 1970-2000 and spatial distribution of key sources in 2000, J. Int. Environ. Sci., 2, 81-99, 2005.

Pacala, S. W., Breidenich, C., Brewer, P. G., Fung, I. Y., Gunson, M.R., Heddle, G., Law, B., Marland, G., Paustian, K., Prather, M., Randerson, J. T., Tans, P., and Wofsy, S. C.: Verifying Greenhouse Gas Emissions: Methods to Support International Climate Agreements, Committee on Methods for Estimating Greenhouse Gas Emissions, National Research Council, The National Academies Press, available at: http://www.nap.edu/ openbook.php?record_id=12883 (last access: 3 February 2014), 2010.

Pregger, T., Scholtz, Y., and Friedrich, R.: Documentation of the anthropogenic GHG emission data for Europe provided in the frame of CarboEurope GHG and CarboEurope IP, Final Report, Tech. rep., Institute of Energy Economics and the Rational Use of Energy, University of Stuttgart, 2007. 
Raupach, M. R., Rayner, P. J., and Page, M.: Regional variations in spatial structure of nightlights, population density and fossil-fuel $\mathrm{CO}_{2}$ emissions, Energ. Policy, 38, 4756-4764, 2009.

Ray, J.: Estimating $\mathrm{ffCO}_{2}$ using a MsRF and sparse reconstruction, available at: http://www.sandia.gov/ jairay/software.html (last access: 3 February 2014), 2013.

Ray, J., Lee, J., Lefantzi, S., Yadav, V., Michalak, A. M., BloemenWaanders, B., and McKenna, S. A.: A multiresolution spatial parametrization for the estimation of fossil-fuel carbon dioxide emissions via atmospheric inversions, SAND Report SAND2013-2919, Sandia National Laboratories, Livermore, CA 94551-0969, available at: http://www.sandia.gov/ jairay (last access: 3 February 2014), also available at https://cfwebprod. sandia.gov/cfdocs/CompResearch/docs/sand2013-2919.pdf (last access: 1 July 2014), 2013.

Rayner, P. J., Raupach, M. R., Paget, M., Peylin, P., and Koffi, E.: A new global gridded data set of $\mathrm{CO}_{2}$ emissions from fossil fuel combustion: methodology and evaluation, J. Geophys. Res., 115, D19306, doi:10.1029/2009JD013439, 2010.

Romberg, J. K., Choi, H., and Baraniuk, R. G.: Bayesian tree-structured image modeling using wavelet-domain Hidden Markov Models, IEEE T. Image Process., 10, 1056-1068, 2001.

Simons, F. J., Loris, I., Nolet, G., Daubechies, I. C., Voronin, S., Judd, J. S., Vetter, P. A., Vetter, P. A., Charlety, J., and Vonesch, C.: Solving or resolving global tomographic models with spherical wavelets and the scale and sparsity of seismic heterogeneity, Geophys. J. Int., 187, 969-988, 2011.

Skamarock, W. C. and Klemp, J. B.: A time-split nonhydrostatic atmospheric model for weather research and forecasting applications, J. Comput. Phys., 227, 3465-3485, 2008.
Strang, G. and Nguyen, T.: Wavelets and Filter Banks, WellesleyCambridge Press, Wellesley, MA, 1997.

Streets, D. G., Zhang, Q., Wang, L., He, K., Hao, J., Wu, Y., Tang, Y., and Carmichael, G. R.: Revisting China's CO emissions after transport and chemical evolution over the Pacific: synthesis of inventories, atmospheric modeling and observations, J. Geophys. Res., 111, D14306, doi:10.1029/2006JD007118, 2006.

Taubman, D. and Marcellin, M.: JPEG2000: Image Compression Fundamentals, Standards and Practice, Springer, 2002.

Tropp, J. and Gilbert, A. C.: Signal recovery from partial information via orthogonal matching pursuit, IEEE T. Inform. Theory, 53, 4655-4666, 2007.

Tsaig, Y. and Donoho, D.: Extensions of compressed sensing, Signal Process., 86, 533-548, 2006.

Turnbull, J. C., Karion, A., Fischer, M. L., Faloona, I., Guilderson, T., Lehman, S. J., Miller, B. R., Miller, J. B., Montzka, S., Sherwood, T., Saripalli, S., Sweeney, C., and Tans, P. P.: Assessment of fossil fuel carbon dioxide and other anthropogenic trace gas emissions from airborne measurements over Sacramento, California in spring 2009, Atmos. Chem. Phys., 11, 705-721, doi:10.5194/acp-11-705-2011, 2011.

Walker, J. S.: A Primer on Wavelets and Their Scientific Applications, Chapman and Hall/CRC, 2008.

Welstead, S.: Fractal and wavelet image compression techniques (SPIE tutorial texts in optical engineering Vol. TT40), SPIE Press, Bellingham, WA USA, 1999.

Williams, J. R. and Amaratunga, K.: Introduction to wavelet engineering, Int. J. Numer. Meth. Eng., 37, 2365-2388, 1994. 